ORNL/TM-12269

Engineering Physics and Mathematics Division

\title{
RARE ATTRIBUTES IN FINITE UNIVERSES: HYPOTHESES TESTING SPECIFICATION AND EXACT RANDOMIZED UPPER CONFIDENCE BOUNDS
}

\author{
Tommy Wright \\ Mathematical Sciences Section \\ Engineering Physics and Mathematics Division \\ Oak Ridge National Laboratory \\ P.O. Box 2008, Building 6012 \\ Oak Ridge, Tennessee 37831-6367
}

Date Published: March, 1993

Research sponsored by the Applied Mathematical Sciences Research Program, Office of Energy Research, U.S. Department of Energy, under contract DE-AC05-84OR21400 with the Martin Marietta Energy Systems, ]nc.

Prepared by the

OAK RIDGE NATIONAL LABORATORY

Oak Ridge, Tennessee 37831

MARTIN MARIETTA ENERGY SYSTEMS, INC.

for the

U.S. DEPARTMENT OF ENERGY under Contract No. DE-AC05-84OR21400

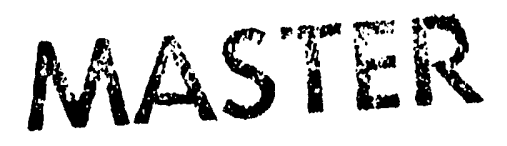




\section{TABLE OF CONTENTS}

LIST OF TABLES

LIST OF FIGURES

ABSTRACT . . . . . . . . . . . . . . . vii

1. INTRODUCTION . . . . . . . . . . . . . . 1

2. BACKGROUND •. . . . . . . . . . . . . . 3

3. TESTING FOR RARE ATTRIBUTES: $A=0$. . . . . . . 7

3.1. Against $A=A_{1}(>0) \quad$. . . . . . . . . . . . . . . 7

3.2. Against $A=A_{1}(=1) \quad$. . . . . . . . . . . . . 10

3.3. Definition of An Optimal Sampling Design When Testing for

Rare Attributes . . . . . . . . . . . . . 11

4. SOME GENERAL RESULTS . . . . . . . . . . . . . 12

5. TESTING $(0,1)$ OR $(1,0)$ UNDER COMPLEX

SAMPLING DESIGNS . . . . . . . . . . . . . . 14

5.1. Testing $(0,1)$ or $(1,0)$ Under Stratified

Random Sampling . . . . . . . . . . . . . . 14

5.2. Testing $(0,1)$ or $(1,0)$ Under Two-Stage

Cluster Sampling

5.2.1. Testing with Random Selection at

Both Stages . . . . . . . . . . . . 15

5.2.2. Testing with Probability Proportional

to Size $(\pi p s)$ Sampling at Stage One . . . . . 17

6. EXACT RANDOMIZED UPPER CONFIDENCE BOUNDS $\cdot 17$

6.1. Definition and Construction Algorithm . . . . . . 19

6.2. Example . . . . . . • . . . . . . . . . 25

7. CONCLUSIONS . . . . . . . . . . . . . 27

ACKNOWLEDGMENTS . . . . . . . . . . . 28

REFERENCES . . . . . . . . . . . . . . . 29 


\section{LIST OF TABLES}

Table 1. Power Comparisons for Testing $(0,1)$ or $(1,0)$ - . . - 18

Table 2. Comparison of Coverage Probabilities for $\hat{A}_{U}(\cdot)$ and $\hat{A}_{R U}(\cdot) 27$

\section{LIST OF FIGURES}

Figure 1. All Possible Values of $\phi_{L, A}(a)$ - . • • • • • • • • • 20

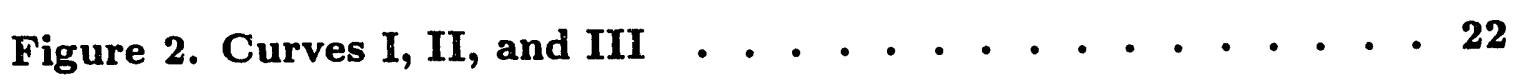

Figure 3. Visualization of an Exact Randomized $100(1-\alpha) \%$ Upper Confidence Bound for $A$ Corresponding to Observed $a \quad{ }^{2}$

Figure 4. Values of $\phi_{L, A}$ for $N=10, n=4$, and $1-\alpha=.975 \quad$. . 26 


\title{
RARE ATTRIBUTES IN FINITE UNIVERSES: HYPOTHESES TESTING SPECIFICATION AND EXACT RANDOMIZED UPPER CONFIDENCE BOUNDS
}

\author{
Tommy Wright
}

\begin{abstract}
When attributes are rare and few or none are observed in the selected sample from a finite universe, sampling statisticians are increasingly being challenged to use whatever methods are available to declare with high probability or confidence that the universe is near or completely attribute-free. This is especially true when the attribute is undesirable. Approximations such as those based on normal theory are frequently inadequate with rare attributes. For simple random sampling without replacement, an appropriate probability distribution for statistical inference is the hypergeometric distribution. But even with the hypergeometric distribution, the investigator is limited from making claims of attribute-free with high confdence unless the sample size is quite large using nonrandomized techniques. In the hypergeometric setting with rare attributes, exact randomized tests of hypothesis are investigated to determine the effect on power of how one specifies the null hypothesis. In particular, specifying the null hypothesis as zero attributes does not always yield maximum possible power. We also consider the hypothesis specification question under complex sampling designs including stratified random sampling and two-stage cluster sampling (one case involves random selection at first stage and another case involves probability proportional to size without replacement selection at first stage). Also under simple random sampling, this article defines and presents a simple algorithm for the construction of exact "randomized" upper confidence bounds which permit one to possibly report tighter bounds than those exact bounds obtained using "nonrandomized" methods.
\end{abstract}

KEY WORDS: Exact randomized $\alpha$-level tests; Exact randomized upper confidence bounds; Hypergeometric; Neyman-Pearson Lemma; Power. 


\section{INTRODUCTION}

Applications abound for sampling from a finite universe of size $N$ to make inferences about the number $A$ with a specific attribute in the universe (Cochran 1977; Hajék 1981; Hald 1981; Hansen, Hurwitz, and Madow 1953; Schilling 1982). Under simple random sampling without replacement, especially in small universes where $A$ is assumed near zero, e.g., sampling inspection and sampling for audit applications, the hypergeometric probability distribution is the appropriate sampling distribution for exact statistical inferences (Johnson and Kotz 1977; Liebermann and Owen 1961; Odeh and Owen 1983; Wright 1991). For any $N$ and when $A$ is at or near zero, e.g. $A=0,1$, or 2 , we say that the associated attribute is a rare attribute.

Increasingly for applications with certain finite universes, investigators are asked to take necessary steps to eventually report with high probability or confidence that $A$ is near or equal to zero without $100 \%$ inspection. Examples of sampling where attributes are rare and where high assurance is required that $A=0$ include: sampling to assure that all welds on a nuclear reactor are properly formed, sampling to assure that all houses in a certain area are below a threshold radon reading, sampling to assure that all valuable materials and parts can be accounted for, and sampling to assure that all drums of waste materials being treated have readings which permit treatment. Most important are those cases where the attribute is a defective or some other undesirable trait. A recent overview of statistical concerns with annotated bibliography when auditing populations assumed to contain rare errors is given in a report of the Panel on Nonstandard Mixtures of Distributions (1989). Hwang (1972) provides a simple group testing method for detecting all defectives in a universe when there is an upper bound on the number of defectives $A$. However, group testing methods have limited applications. Wright (1990) shows under simple random sampling without replacement that if one is to be $100(1-\alpha) \%$ confident that there are no defective units in the universe given that no defectives were observed in the sample, then the sampling fraction must be at least $1-\alpha$, i.e., we must have $n / N \geq 1-\alpha$. When $1-\alpha$ is near 1 , the required sampling fractions and costs are high, and alternative methods are needed. When there is strong prior information, Wright (1992) provides an alternative which gives an explicit decision rule in a Bayesian context for answering the question: "What is the maximum number of units in the universe that need not be inspected, if the 
posterior probability that none of these are defective must be greater than or equal to $1-\epsilon$ for $0<\epsilon<1$ ?" With this Bayesian method and depending on the value of $N$, the savings in terms of a sampling effort rather than a census can be significant.

In a hypothesis testing approach for rare attributes, one wants to test whether or not $A=0$. Indeed, the decision is often between only two values $A=0$ and $A=A_{1}$ where $0<A_{1} \leq N$. When the choice is between two values, the null hypothesis is often taken as

$$
H_{0}: A=0
$$

and the alternative hypothesis is stated as

$$
H_{1}: A=A_{1}
$$

(The more familiar form in practice is $H_{0}: A=0$ vs. $H_{1}: A \geq A_{1}$.) However, it is worth noting that taking the null hypothesis to be $A=0$ when $A=0$ is desired to be supported by the sample data is contrary to the usual practice of stating the desired value(s) of the parameter to be supported by the data under the alternative hypothesis.

If one knows that the true value of $A$ is either 0 or $A_{1}$ and not both, an answer of yes to the question, "Is it true that $A=0$ ?" is correct if and only if the correct answer is no to the question "Is it true that $A=A_{1}$ ?" and vice versa. However, and contrary to the intuition of many, testing $A=0$ against $A=A_{1}$ is not equivalent to the specification of testing $A=A_{1}$ against $A=0$. While this fact that testing with one specified order is not equivalent to testing with another specified order is noted and accepted by statisticians without any apparent feeling of discomfort (see e.g., Bunt and Barton, 1967, pp. 128-9), the same cannot be said of nonstatisticians who are the majority users of statistical methodology which purports to being useful in dealing with uncertainty (Barabba, 1991).

Desiring that the probabilities of Type I and Type II errors be as small as possible given the serious consequences of error and the budget constraints, the investigator will want to maximize the power when specifying which of $A=0$ or $A=A_{1}$ should be the null hypothesis and which should be the alternative hypothesis. This article investigates and presents exact results using the hypergeometric distribution for testing one simple hypothesis against another simple hypothesis demonstrating explicitly the effect that specification of hypotheses has on power. For completeness, Section 2 reviews the basic theory of Neyman and Pearson (1933) 
for testing two simple hypotheses in the finite universe context with exact significance level $\alpha$. In Section 3, we consider the effect on power of order specification when testing $A=0$ against $A=A_{1}$ where $A_{1}$ is a particular positive integer; a special case of interest is $A_{1}=1$. In Section 4, we present conditions for a test of power 1 under the alternative and a general result when specification order has no effect on power. The problem of testing for rare attributes when $A_{1}=1$ is considered under more complex sampling strategies including: stratified random sampling, two-stage cluster sampling, and probability proportional to size sampling without replacement in Section 5. Exact randomized upper confidence bounds are defined and constructed in Section 6. Concluding remarks are given in Section 7.

\section{BACKGROUND}

For a finite universe of size $N$ with an unknown number of units $A$ having a particular attribute, let $A_{0}$ and $A_{1}$ be two different specified integers (i.e. either $A_{0}<A_{1}$ or $\left.A_{0}>A_{1}\right)$ such that $0 \leq A_{0}, A_{1} \leq N$. Assume that a simple random sample without replacement of size $n$ is to be selected to test at stated significance level $\alpha(0<\alpha<1)$ the hypotheses

$$
H_{0}: A=A_{0} \text { vs. } H_{1}: A=A_{1} \text {. }
$$

If $\Omega_{A}$ is the set of feasible values of $A$, (3) implies that $\Omega_{A}=\left\{A_{0}, A_{1}\right\}$. Let $\boldsymbol{a}$ be the random variable representing the number of sample units with the attribute. The sampling distribution of $\boldsymbol{a}$ under simple random sampling without replacement is hypergeometric as given in (4)

$$
p(\boldsymbol{a}=a \mid A)=\frac{\left(\begin{array}{c}
A \\
a
\end{array}\right)\left(\begin{array}{c}
N-A \\
n-a
\end{array}\right)}{\left(\begin{array}{l}
N \\
n
\end{array}\right)} \text { for } \max (0, n-N+A) \leq a \leq \min (A, n) .
$$

For simplicity, we declare the support of $a$ to be $\{0,1,2, \ldots, n\}$ and assign zero probabilities for those values of $a$ where appropriate. With rare attributes, we will always have $A$ being much smaller than $n$ in practice.

The critical region $C$ consists of those values of $a$ which lead to rejection of $H_{0}$, while $C^{\prime}$ consists of those values of $a$ which lead to nonrejection of $H_{0}$. The set $C$ is often referred to as the test of $H_{0}$ against $H_{1}$. The probability of rejecting $H_{0}$ 
given a specific value of $A$ by using the critical region $C$, written $P(C \mid A)$, is called the power of the test $C$ for the value $A$. Clearly,

$$
P(C \mid A)=\sum_{a \in C} p(a \mid A)
$$

Most interest is in evaluating (5) when $A$ is a value specified under the alternative hypothesis. Because $H_{1}: A=A_{1}$ is a simple hypothesis, the power of the test in (3) is given by $P\left(C \mid A_{1}\right)$. The power under $H_{0}: A=A_{0}$ is $P\left(C \mid A_{0}\right)=\alpha$, the significance level of the test where $0<\alpha<1$. To reject $H_{0}$ when $H_{0}$ is true is called a Type I error, while not rejecting $H_{0}$ when $H_{0}$ is not true is called a Type II error. Thus, $P$ (Type I error $)=P\left(C \mid A_{0}\right)=\alpha$, and $P$ (Type II error) $=1-P\left(C \mid A_{1}\right)=\beta$, where $0<\beta<1$ for testing (3).

$A$ set $C$ is a most powerful test (equivalently a most powerful critical region) for testing the hypotheses in (3) at significance level $\alpha$ if (i) $P\left(C \mid A_{0}\right)=\alpha$ and (ii) if $D$ is any other subset of $\{0,1,2, \ldots, n\}$ such that $P\left(D \mid A_{0}\right) \leq \alpha$, then $P\left(C \mid A_{1}\right) \geq$ $P\left(D \mid A_{1}\right)$. The following lemma, which is a special case of the Neyman-Pearson Lemma, gives conditions under which a most powerful test (critical region) can always be found when testing the hypotheses in (3).

N-P Lemma (1999). Let $a$ be the random variable representing the number of units with a particular attribute in a simple random sample without replacement of size $n$ selected from a finite universe of $N$ units. Let $A_{0}$ and $A_{1}$ be two different specified integers where $0 \leq A_{0}, A_{1} \leq N$. Let $0<\alpha<1$ be fixed. Let $k$ be a positive real number and $C$ be a subset of the sample space $\{0,1,2, \ldots, n\}$ where $k$ and $C$ satisfy:

$$
\begin{array}{ll}
\text { (i) } P\left(C \mid A_{0}\right)=\alpha & \\
\text { (ii) } p\left(a \mid A_{1}\right) / p\left(a \mid A_{0}\right) \geq k \quad \text { if } & a \in C \text { and } \\
\text { (iii) } p\left(a \mid A_{1}\right) / p\left(a \mid A_{0}\right)<k \quad \text { if } & a \in C^{\prime} .
\end{array}
$$

Then $C$ is a most powerful test of significance level $\alpha$ for testing $H_{0}: A=A_{0}$ against $H_{1}: A=A_{1}$.

The N-P Lemma makes it clear that values of $a$ with high ratio values $p\left(a \mid A_{1}\right) / p\left(a \mid A_{0}\right)$ favor rejection of $H_{0}$ where high is expressed in terms of $k$. For implementation of this lemma in practice, the following results are helpful. In each case, we assume simple random sampling without replacement from a finite universe of size $N$ where $a$ is the number of attributes in the sample of size $n$. 
Lemma 1. For given values $a, k$, and $A_{0}(>0)$, there exists $k^{*}$ such that

$$
p\left(a \mid A_{0}-1\right) / p\left(a \mid A_{0}\right) \geq k \text { iff } a \leq k^{*}
$$

where $k^{*}$ depends on $k$.

Proof:

$$
\begin{gathered}
p\left(a \mid A_{0}-1\right) / p\left(a \mid A_{0}\right) \geq k \text { iff }\left(\begin{array}{c}
A_{0}-1 \\
a
\end{array}\right)\left(\begin{array}{c}
N-A_{0}+1 \\
n-a
\end{array}\right) /\left[\left(\begin{array}{c}
A_{0} \\
a
\end{array}\right)\left(\begin{array}{c}
N-A_{0} \\
n-a
\end{array}\right)\right] \geq k \\
\quad \text { iff } a \leq \frac{A_{0}\left(N-A_{0}+1\right)-k A_{0}\left(N-A_{0}+1-n\right)}{\left(N-A_{0}+1+k A_{0}\right)}
\end{gathered}
$$

iff $a \leq k^{*}$

where $k^{*}=\frac{A_{0}\left(N-A_{0}+1\right)-k A_{0}\left(N-A_{0}+1-n\right)}{\left(N-A_{0}+1+k A_{1}\right)}$.

The importance of Lemma 1 is that for testing $H_{0}: A=A_{0}$ vs. $H_{1}: A=A_{0}-1$, the most powerful test $C$ for given $\alpha$ which (by the N-P Lemma) rejects $H_{0}$ for all $a$ such that $p\left(a \mid A_{0}-1\right) / p\left(a \mid A_{0}\right) \geq k$ is equivalent to the most povirful test which rejects $H_{0}$ for all $a$ such that $a \leq k^{*}$, i.e., rejects $H_{0}$ for small values of $a$. The obvious advantage is that $k^{*}$ can be easily found to satisfy $P\left(a \leq k^{*} \mid A_{0}\right)=\alpha$ by making use of (4). Lemma 1 leads to the following important corollary.

Corollary 1. For given values $a$ and $k$ and $A_{0}>A_{1}$, a most powerful test of significance level $\alpha$ for testing $H_{0}: A=A_{0}$ vs. $H_{1}: A=A_{1}$ is to reject $H_{0}$ in favor of $H_{1}$ if $a \leq k^{*}$ where $k^{*}$ is chosen to satisfy $P\left(a \leq k^{*} \mid A_{0}\right)=\alpha$. (When $A_{1}=A_{0}-1, k^{*}$ is given explicitly as noted in the proof of Lemma 1.)

In a similar way, Lemma 2 implies that for testing $H_{0}: A=A_{0}$ vs. $H_{1}: A=$ $A_{0}+1$, a most powerful test $C$ which rejects $H_{0}$ for all $a$ such that $p\left(a \mid A_{0}+\right.$ 1) $/ p\left(a \mid A_{0}\right) \geq k$ is equivalent to the most powerful test which rejects $H_{0}$ for all $a$ such that $a \geq k^{* *}$, i.e., rejects $H_{0}$ for large values of $a$.

Lemma 2. For given values $a, k$, and $A_{0}$, there exists $k^{* *}$ such that

$$
p\left(a \mid A_{0}+1\right) / p\left(a \mid A_{0}\right) \geq k \quad \text { iff } a \geq k^{* *}
$$

where $k^{* *}$ depends on $k$ (Lehmann $1959 ;$ p. 70$)$. 
Thus, Corollary 2 follows as did Corollary 1.

Corollary 2. For given values $a$ and $k$ and $A_{0}<A_{1}$, a most powerful test of significance level $\alpha$ for testing $H_{0}: A=A_{0}$ vs. $H_{1}: A=A_{1}$ is to reject $H_{0}$ in favor of $H_{1}$ if $a \geq k^{* *}$ where $k^{* *}$ is chosen to satisfy $P\left(a \geq k^{* * *} \mid A_{0}\right)=\alpha$.

Comment. Because the sampling distribution of $a$ is discrete, it is not always possible to find a $k>0$ and critical region $C$ which satisfy the assumptions of the $N-P$ Lemma exactly for a given value of $\alpha$. In practice, two options are considered.

Option I. Change the value of $\alpha$ to $\tilde{\alpha}$. Take $\tilde{\alpha}$ to be some number less (or more) than the originally stated $\alpha$ so that for $\tilde{\alpha}$, a $\tilde{k}$ exists which gives a critical region with significance level equal to $\tilde{\alpha}$.

While Option I is the option chosen most often in practice, Option II is presented next to demonstrate how one can construct a (most powerful) test where the level of significance is exactly $\alpha$ and to serve as a reference throughout the remainder of this paper.

Option II. Use a randomized test $\phi$. The tests discussed thus far in this paper are nonrandomized tests because each $a$ is clearly specified to either belong to $C$ or $C^{\prime}$. Sometimes, the assignment of a particular value of $a$ to $C$ or $C^{\prime}$ is unclear or not as sharp as desired. One such case occurs with randomized tests.

Let $\phi(a)$ be the probability of rejecting $H_{0}$ if $a$ is observed. To test $H_{0}: A=A_{0}$ vs. $H_{1}: A=A_{1}$ where $A_{1}>A_{0}$, the randomized test (or critical function) $\phi_{U}$ is given by (subscript " $U$ " refers to upper tail critical region)

$$
\phi_{U}(a)= \begin{cases}1 & \text { if } a=a_{U}+1, a_{U}+2, \ldots, n-1, n \\ \frac{\alpha-\sum_{a=a_{U}+1}^{n} p\left(a \mid A_{0}\right)}{p\left(a_{U} \mid A_{0}\right)} & \text { if } a=a_{U} \\ 0 & \text { if } a=0,1,2, \ldots, a_{U}-1 .\end{cases}
$$

where $a_{U}$ is such that $\sum_{a=a_{U}+1}^{n} p\left(a \mid A_{0}\right) \leq \alpha$ and $\sum_{a=a_{U}}^{n} p\left(a \mid A_{0}\right)>\alpha$. The significance level of $\phi_{U}$ is $E_{A_{0}}\left[\phi_{U}(a)\right]=\sum_{a=0}^{n} \phi_{U}(a) p\left(a \mid A_{0}\right)=\alpha$, and the power of $\phi_{U}$ under $A_{1}$ is $E_{A_{1}}\left[\phi_{U}(a)\right]=\sum_{a=0}^{n} \phi_{U}(a) p\left(a \mid A_{1}\right)$. The critical region $C_{U}$ corresponding to the randomized test $\phi_{U}$ can be viewed as a fuzzy set (Zadeh 1965) $C_{U}=\left\{a_{U}, a_{U}+1, \ldots, n-1, n\right\}$ where each of $a_{U}+1, a_{U}+2, \ldots, n-1, n$ belongs to $C_{U}$ with degree $\phi_{U}(a)=1$, while $a_{U}$ belongs to $C_{U}$ with degree $\phi_{U}\left(a_{U}\right)<1$. Here 
the function $\phi_{U}$ is viewed as the degree of membership function. In the context of fuzzy sets and only if $a_{U}$ units with the attribute are observed in the sample, the sample evidence is indecisive and randomization (i.e. a Bernoulli trial) is employed to reach a final decision to either reject $H_{0}$ with probability $\phi_{U}\left(a_{U}\right)$ or not to reject $H_{0}$ with probability $1-\phi_{U}\left(a_{U}\right)$.

Similarly, to test $H_{0}: A=A_{0}$ vs. $H_{1}: A=A_{1}$ where $A_{1}<A_{0}$, the randomized test (or critical function) $\phi_{L}$ is given by (subscript " $L$ " refers to lower tail critical region)

$$
\phi_{L}(a)= \begin{cases}1 & \text { if } a=0,1,2, \ldots, a_{L}-1 \\ \frac{\alpha-\sum_{a=0}^{a_{L}-1} p\left(a \mid A_{0}\right)}{p\left(a_{L} \mid A_{0}\right)} & \text { if } a=a_{L} \\ 0 & \text { if } a=a_{L}+1, a_{L}+2, \ldots, n-1, n\end{cases}
$$

where $a_{L}$ is such that $\sum_{a=0}^{a_{L}-1} p\left(a \mid A_{0}\right) \leq \alpha$ and $\sum_{a=0}^{a_{L}} p\left(a \mid A_{0}\right)>\alpha$. The significance level of $\phi_{L}$ is $E_{A_{0}}\left[\phi_{L}(\boldsymbol{a})\right]=\alpha$, and the power of $\phi_{L}$ under $A_{1}$ is $E_{A_{1}}\left[\phi_{L}(\boldsymbol{a})\right]$. As for $C_{U}$, the critical region $C_{L}=\left\{0,1,2, \ldots, a_{L}-1, a_{L}\right\}$ corresponding to $\dot{\phi}_{L}$ can be viewed as a fuzzy set.

To demonstrate the explicit effect on power due to the specification of hypotheses, randomized tests are essential to ensure the existence of exact $\alpha$-level tests under the two specifications of the null hypothesis, i.e., for $H_{0}: A=A_{0}$ and for $H_{0}: A=A_{1}$.

\section{TESTING FOR RARE ATTRIBUTES: $A=0$}

\subsection{Against $A=A_{1}(>0)$.}

Particularly in applications where efforts have been undertaken to ensure that $A=0$ for a given finite universe, the null hypothesis and alternative hypothesis are often taken as

$$
H_{0}: A=0 \text { vs. } H_{1}: A=A_{1}
$$

which we will denote by the ordered pair $\left(0, A_{1}\right)$. Alternatively, the ordered pair $\left(A_{1}, 0\right)$ will denote

$$
H_{0}: A=A_{1} \text { vs. } H_{1}: A=0 \text {. }
$$

In general, the first position of the ordered pair specifies the null hypothesis, while the second position specifies the alternative hypothesis. Here, $\Omega_{A}=\left\{0, A_{1}\right\}$. 
More importantly than rejecting $H_{0}: A=0$ in (8) or rejecting $H_{0}: A=A_{1}$ in (9), the experimenter wants to know which one of the two values in $\Omega_{A}$ is the truth. It is desirable that the order $\left(0, A_{1}\right)$ or $\left(A_{1}, 0\right)$ should not matter; but it is well known that order does matter in the usual statistical approach and Lemmas 3, 4, and 5 illustrate just how explicitly. Let $\operatorname{POWER}\left(A_{1}, 0\right)$ and $\operatorname{POWER}\left(0, A_{1}\right)$ represent the power under the alternatives for testing $\left(A_{1}, 0\right)$ and $\left(0, A_{1}\right)$, respectively.

Lemma 9. Let $0<\alpha<1,1 \leq A_{1}<N$, and $0<p\left(a=0 \mid A_{1}\right) \leq \alpha$. Then (i) testing with order $\left(A_{1}, 0\right)$ has $\operatorname{POWER}\left(A_{1}, 0\right)=1$, and (ii) testing with order $\left(0, A_{1}\right)$ has POWER $\left(0, A_{1}\right)<1$.

Proof: (i) Testing with order $\left(A_{1}, 0\right)$, the randomized test $\phi_{L}$ is given by

$$
\phi_{L}(a)= \begin{cases}1 & \text { if } a=0,1, \ldots, a_{L}-1 \\ \frac{\alpha-\sum_{a=0}^{a_{L}-1} p\left(a \mid A_{1}\right)}{p\left(a_{L} \mid A_{1}\right)} & \text { if } a=a_{L} \\ 0 & \text { if } a=a_{L}+1, a_{L}+2, \ldots, n-1, n,\end{cases}
$$

where $a_{L}(\geq 1)$ is such that $\sum_{a=0}^{a_{L}-1} p\left(a \mid A_{1}\right) \leq \alpha$ and $\sum_{a=0}^{a_{L}} p\left(a \mid A_{1}\right)>\alpha$. For $\phi_{L}$, $P\left(C_{L} \mid A=A_{1}\right)=E_{A_{1}}\left[\phi_{L}(a)\right]=\alpha$, and the power under $A=0$ is

$$
\begin{aligned}
\operatorname{POWER}\left(A_{1}, 0\right)=P\left(C_{L} \mid A=0\right) & =E_{0}\left[\phi_{L}(a)\right] \\
& =\sum_{a=0}^{a_{L}-1} \phi_{L}(a) p(a \mid A=0)+\phi_{L}\left(a_{L}\right) p\left(a_{L} \mid A=0\right) \\
& =1+\phi_{L}\left(a_{L}\right) \cdot(0)=1 .
\end{aligned}
$$

Comment. If $p\left(a=0 \mid A_{1}\right)<\alpha$, then $\phi_{L}\left(a_{L}\right)>0$ for some $a_{L} \geq 1$. Thus, when $p\left(a=0 \mid A_{1}\right)<\alpha$ and because any observed value of $a>0$ cannot logically support $H_{1}: A=0$, we should take a new significance level as suggested under Option I, say $\tilde{\alpha}$, such that $\tilde{\alpha}=p\left(a=0 \mid A_{1}\right)$. This will ensure that a revised test $\tilde{\phi}_{L}$ will have $\tilde{\phi}_{L}\left(a_{L}\right)=0$ for $a_{L} \geq 1$ and that $\tilde{\phi}_{L}$ is a nonrandomized test. The proof of (ii) is next.

(ii) Testing with order $\left(0, A_{1}\right)$, the randomized test $\phi_{U}$ is given by

$$
\phi_{U}(a)= \begin{cases}1 & \text { if } a=1,2, \ldots, n \\ \alpha & \text { if } a=0 .\end{cases}
$$


Here, $P\left(C_{U} \mid A=0\right)=E_{0}\left[\phi_{U}(a)\right]=\alpha$, and the power of $\phi_{U}$ for $A_{1}$ is $\operatorname{POWER}\left(0, A_{1}\right)=P\left(C_{U} \mid A=A_{1}\right)=E_{A_{1}}\left[\phi_{U}(a)\right]=1-p\left(0 \mid A_{1}\right)(1-\alpha)<1$.

From Lemma 3 and if $p\left(a=0 \mid A_{1}\right)<\alpha$, the significance level should be reduced to a new level $\tilde{\alpha}=p\left(a=0 \mid A_{1}\right)$. Secondly, for $p\left(a=0 \mid A_{1}\right)=\alpha$, testing with order $\left(A_{1}, 0\right)$ where $A_{1}$ is specified as the null hypothesis yields power equal to 1 , and it will always be superior to testing with order $\left(0, A_{1}\right)$ where 0 is specified as the null hypothesis and the power is less than 1 . In Lemma 4 , we consider $\left(0, A_{1}\right)$ comparud with $\left(A_{1}, 0\right)$ when $p\left(a=0 \mid A_{1}\right)>\alpha$.

Lemma 4. Let $0<\alpha<1,1 \leq A_{1}<N$, and $p\left(\alpha=0 \mid A_{1}\right)>\alpha$.

(i) If $\alpha>p\left(a=0 \mid A_{1}\right) /\left\{1+p\left(a=0 \mid A_{1}\right)\right\}$, then $\operatorname{POWER}\left(A_{1}, 0\right)>\operatorname{POWER}\left(0, A_{1}\right)$.

(ii) If $\alpha<p\left(a=0 \mid A_{1}\right) /\left\{1+p\left(a=0 \mid A_{1}\right)\right\}$, then $\operatorname{POWER}\left(A_{1}, 0\right)<\operatorname{POWER}\left(0, A_{1}\right)$.

(iii) If $\alpha=p\left(a=0 \mid A_{1}\right) /\left\{1+p\left(a=0 \mid A_{1}\right)\right\}$, then $\operatorname{POWER}\left(A_{1}, 0\right)=\operatorname{POWER}\left(0, A_{1}\right)$.

Proof: (i) Testing with order $\left(A_{1}, 0\right)$, the randomized test is

$$
\phi_{L}(a)= \begin{cases}\alpha / p\left(a=0 \mid A_{1}\right) & \text { if } a=0 \\ 0 & \text { if } a=1,2, \ldots, n .\end{cases}
$$

where $P\left(C_{L} \mid A=A_{1}\right)=\phi_{L}(0) p\left(\boldsymbol{a}=0 ; A_{1}\right)=\alpha$ and $\operatorname{POWER}\left(A_{1}, 0\right)=P\left(C_{L} \mid A=\right.$ $0)=\phi_{L}(0) p(a=0 \mid A=0)=\alpha / p\left(a=0 \mid A_{1}\right)$. Also, testing with order $\left(0, A_{1}\right)$, the randomized test is

$$
\phi_{U}(a)= \begin{cases}1 & \text { if } a=1,2,3, \ldots, n \\ \alpha & \text { if } a=0 .\end{cases}
$$

where $P\left(C_{U} \mid A=0\right)=\phi_{U}(0) p(a=0 \mid A=0)=\alpha$ and $\operatorname{POWER}\left(0, A_{1}\right)=P\left(C_{U} \mid A=\right.$ $\left.A_{1}\right)=\sum_{a=0}^{n} \phi_{U}(a) p\left(a \mid A_{1}\right)=1-p\left(a=0 \mid A_{1}\right)+p\left(a=0 \mid A_{1}\right) \alpha$. Now if

$$
\begin{gathered}
\alpha>p\left(\boldsymbol{a}=0 \mid A_{1}\right) /\left\{1+p\left(\boldsymbol{a}=0 \mid A_{1}\right)\right\} \\
\alpha\left\{1-\left[p\left(\boldsymbol{a}=0 \mid A_{1}\right)\right]^{2}\right\} / p\left(\boldsymbol{a}=0 \mid A_{1}\right)>1-p\left(\boldsymbol{a}=0 \mid A_{1}\right), \\
\alpha / p\left(\boldsymbol{a}=0 \mid A_{1}\right)>1-p\left(\boldsymbol{a}=0 \mid A_{1}\right)+p\left(\boldsymbol{a}=0 \mid A_{1}\right) \alpha \\
\operatorname{POWER}\left(A_{1}, 0\right)>\operatorname{POWER}\left(0, A_{1}\right),
\end{gathered}
$$

and (i) follows. Proofs for (ii) and (iii) are similar.

Comment. It is worth noting that when $\alpha \leq p\left(a=0 \mid A_{1}\right), \phi_{L}(a)=0$ and $\phi_{U}(a)=1$ for $a=1,2, \ldots, n$ while $0<\phi_{U}(0)<\phi_{L}(0)<1$. Thus, both tests cause 
the experimenter to come to similar conclusions if $a=1,2, \ldots, n$ and to randomize if $a=0$. This characteristic is desirable.

\subsection{Against $A=A_{1}(=1)$}

Of particular interest is the case when $A_{1}=1$. (In practice, this often appears in the equivalent [for testing] form $H_{0}: A=0$ vs. $H_{1}: A>0$.) When $A_{1}=1$, $p(a=0 \mid A=1)=(N-n) / N$; and as observed from Lemmas 3 and 4 , it is enough to consider the cases where $\alpha \leq(N-n) / N$.

When $\alpha=(N-n) / N$, Lemma 3 reveals that testing with specified order $(1,0)$ has power 1 and is superior to testing with order $(0,1)$. The cost of this test with power 1 can be expensive, i.e., it requires that $n / N \geq 1-\alpha$ which is the same cost if one wants to declare with confidence $1-\alpha$ that 0 is an upper confidence bound for $A$ having observed 0 attributes in the sample (Wright 1990). This same cost also follows from a sequential sampling plan of Wallenius (1967). It has also been noted in a Bayesian setting with a uniform prior on $A$, that the conditional probability that $A=0$ given $a=0$ attributes are observed in the sample is $(n+1) /(N+1)$, (Benedict i2:20; Wright 1992). Note from (10) that testing with specified order $(0,1)$ has power 1 when $n=N$, i.e., when there is $100 \%$ sampling.

When $\alpha<(N-n) / N, p(a=0 \mid A=1) /\{1+p(a=0 \mid A=1)\}=(N-n) /(2 N-n)$ and the results of Lemma 4 follow by comparing $\alpha$ to $(N-n) /(2 N-n)$.

More generally, and when $\alpha \leq(N-n) / N$, to achieve a minimum power of $1-\beta$ for testing $(0,1)$ requires that

$$
n \geq\left(1-\frac{\beta}{1-\alpha}\right) N,
$$

while to achieve a minimum power of $1-\beta$ for testing $(1,0)$ requires that

$$
n \geq\left(1-\frac{\alpha}{1-\beta}\right) N
$$

Lemma 5. Let $\beta=\alpha \leq(N-n) / N$. If $n=\left(1-\frac{\beta}{1-\alpha}\right) N$, then $\operatorname{POWER}(0,1)=$ $\operatorname{POWER}(1,0)$.

Proof: The result follows by noting that POWER $(0,1)=\alpha+(1-\alpha) \frac{n}{N}$ and that $\operatorname{POWER}(1,0)=N \alpha /(N-n)$. 


\subsection{Definition of An Optimal Sampling Design When Testing for Rare Attributés.}

Thus far in this article, the sampling design has been simple random sampling without replacement. In a finite universe with rare attributes, j.e., $A$ is at or near 0 , the desire is to use a best sampling design to select a sample without replacement of size $n$ to test either $(0,1)$ or $(1,0)$. Assuming $\Omega_{A}=\{0,1\}$, what sampling design should be used? In this subsection, it is argued why simple random sampling without replacement is an example of what will be considered as an optimal sampling design in a context with rare attributes.

Let be the number of attributes in the sample, however chosen. If $A=0$, then $p(a=0 \mid A=0)=1$ for any sampling design. Note here that $p(\cdot)$, which depends on the sampling design, is not necessarily the same as given in (4). If $A=1$, then $p(a=0 \mid A=1)+p(a=1 \mid A=1)=1$, where $p(a=0 \mid A=1)$ depends on the sampling design. When testing $(0,1), \operatorname{POWER}(0,1)=\alpha+(1-\alpha) p(a=1 \mid A=1)$; while testing $(1,0)$ gives $\operatorname{POWER}(1,0)=\alpha / p(a=0 \mid A=1)$ where $\alpha \leq p(a=0 \mid A=$ 1).

With regard to power and for given $\alpha$, the question is how should one select the sample in order to maximize the power of either $(0,1)$ or $(1,0)$ ? In view of POWER $(0,1)$, one should sample so that $p(a=1 \mid A=1)$ is large; and from POWER $(1,0)$, one should sample so that $p(a=0 \mid A=1)$ is small. Both are consistent with intuition. But, with the restriction $\alpha \leq p(a=0 \mid A=1), p(a=0 \mid A=1)$ can be no smaller than $\alpha$. Thus, one should sample so that $p(a=0 \mid A=1)=\alpha$, and the best power values are $\operatorname{POWER}(1,0)=1$ and $\operatorname{POWER}(0,1)=1-\alpha+\alpha^{2}$.

As implied earlier, the power is influenced by $p(a=1 \mid A=1)$ which depends on the sampling design. When one further assumes that the experimenter has absolutely no idea which one of the $N$ units would have the attribute if $A=1$, then clearly there is no reason to favor one particular unit's inclusion in the sample over that of another. In such cases, it is appropriate "to assign roughly the same probability to every possible (unit)" for inclusion in the sample (Raiffa and Schlaifer, 1961, p. 63). Thus, an optimal sampling design when testing for rare attributes with no prior knowledge concerning which unit, if any, might have the attribut would be a noninformative sampling design (Cassel, Särndal, and Wretman 1977; Chaudhuri and Vos 1988). Because without replacement sampling requires $\sum_{i=1}^{N} P\left(U_{i}\right.$ selected $)$ $=n$ and probability sampling requires that $P\left(U_{i}\right.$ selected $)>0$ for each $i$, it follows that an optimal sampling design must be one such that $P\left(U_{i}\right.$ selected $)=n / N$ for 
each $i$. Clearly, simple random sampling without replacement is one example of an optimal sampling design. Thus, when $\Omega_{A}=\{0,1\}$, any sampling design with $P\left(U_{i}\right.$ selected $)=p(a=1 \mid A=1)=n / N$ is optimal.

\section{SOME GENERAL RESULTS}

The next lemma is a generalization of Lemma 3. Note that the case $\sum_{a=0}^{A_{0}} p\left(a \mid A_{1}\right)<\alpha$ should not be considered. The sampling design throughout this section is simple random sampling without replacement.

Lemma 6. If $0<\alpha<1,0 \leq A_{0}<A_{1}<N-A_{0} \leq N$, and $\sum_{a=0}^{A_{0}} p\left(a \mid A_{1}\right)=\alpha$, then $\operatorname{POWER}\left(A_{1}, A_{0}\right)=1$.

Proof: Testing with specified order $\left(A_{1}, A_{0}\right)$, a most powerful nonrandomized test is

$$
\phi_{L}(a)= \begin{cases}1 & \text { if } a=0,1,2, \ldots, A_{0} \\ 0 & \text { if } a=A_{0}+1, A_{0}+2, \ldots, n-1, n\end{cases}
$$

where $C_{L}=\left\{0,1,2, \ldots, A_{0}\right\}$. Clearly, $P\left(C_{L} \mid A_{1}\right)=\alpha$, and the power of $\phi_{L}$ for $A=$ $A_{0}$ is $\operatorname{POWER}\left(A_{1}, A_{0}\right)=P\left(C_{L} \mid A_{0}\right)=\sum_{a=0}^{n} \phi_{L}(a) p\left(a \mid A_{0}\right)=\sum_{a=0}^{A_{0}} p\left(a \mid A_{0}\right)=1$.

In addition to Lemma 4(iii) and Lemma 5, the next result gives conditions where the order specification of the hypotheses makes no difference in powerindependently of the value of $\alpha$ and the value of $n$.

Lemma 7. $\operatorname{POWER}\left(A_{0}, N-A_{0}\right)=\operatorname{POWER}\left(N-A_{0}, A_{0}\right)$

Proof: Without loss of generality, assume that $A_{0}<N-A_{0}$. Let $\alpha$ be given. A most powerful $\alpha$-level test for $\left(A_{0}, N-A_{0}\right)$ is given by

$$
\phi_{U}(a)= \begin{cases}1 & \text { if } a=a_{U}+1, a_{U}+2, \ldots, n \\ \frac{\alpha-\sum_{a=a_{U}+1}^{n} p\left(a \mid A_{0}\right)}{p\left(a_{U} \mid A_{0}\right)} & \text { if } a=a_{U} \\ 0 & \text { if } a=0,1,2, \ldots, a_{U}-1\end{cases}
$$


where $a_{U}$ is such that $\sum_{a=a_{U}+1}^{n} p\left(a \mid A_{0}\right) \leq \alpha$ and $\sum_{a=a_{U}}^{n} p\left(a \mid A_{0}\right)>\alpha$, and $\phi_{U}$ has power for $A=N-A_{0}$ of

$$
\begin{aligned}
\operatorname{POWER}\left(A_{0}, N-A_{0}\right) & =P\left(C_{U} \mid N-A_{0}\right) \\
& =E_{N-A_{0}}\left[\phi_{U}(\boldsymbol{a})\right] \\
& =\sum_{a=a_{U}+1}^{n} p\left(a \mid N-A_{0}\right)+\phi_{U}\left(a_{U}\right) p\left(a_{U} \mid N-A_{0}\right) .
\end{aligned}
$$

A most powerful $\alpha$-level test for $\left(N-A_{0}, A_{0}\right)$ is given by

$$
\phi_{L}(a)= \begin{cases}1 & \text { if } a=0,1,2, \ldots, a_{L}-1 \\ \frac{\alpha-\sum_{a=0}^{a_{L}-1} p\left(a \mid N-A_{0}\right)}{p\left(a_{L} \mid N-A_{0}\right)} & \text { if } a=a_{L} \\ 0 & \text { if } a=a_{L}+1, a_{L}+2, \ldots, n-1, n,\end{cases}
$$

where $a_{L}$ is such that $\sum_{a=0}^{a_{L}-1} p\left(a \mid N-A_{0}\right) \leq \alpha$ and $\sum_{a=0}^{a_{L}} p\left(a \mid N-A_{0}\right)>\alpha$, and $\phi_{L}$ has power for $A=A_{0}$ of

$$
\begin{aligned}
\operatorname{POWER}\left(N-A_{0}, A_{0}\right) & =P\left(C_{L} \mid A_{0}\right) \\
& =E_{A_{0}}\left[\phi_{L}(\boldsymbol{a})\right] \\
& =\sum_{a=0}^{a_{L}-1} p\left(a \mid A_{0}\right)+\phi_{L}\left(a_{L}\right) p\left(a_{L} \mid A_{0}\right) .
\end{aligned}
$$

Because $p\left(a \mid A_{0}\right)=p\left(n-a \mid N-A_{0}\right)$ for the hypergeometric family, we have

$$
\begin{aligned}
\sum_{a=0}^{a_{L}-1} p\left(a \mid A_{0}\right) & =\sum_{a=a_{U}+1}^{n} p\left(a \mid N-A_{0}\right) ; \\
p\left(a_{L} \mid A_{0}\right) & =p\left(a_{U} \mid N-A_{0}\right) ; \text { and } \\
p\left(a_{L} \mid N-A_{0}\right) & =p\left(a_{U} \mid A_{0}\right) .
\end{aligned}
$$

Thus, POWER $\left(A_{0}, N-A_{0}\right)=\operatorname{POWER}\left(N-A_{0}, A_{0}\right)$, and ordering of the hypotheses makes no difference.

Comment. A result analogous to Lemma 7 holds for testing the Bernoulli parameter $p$ against $1-p$, or equivalently for testing $A_{0}$ against $N-A_{0}$ under simple random sampling with replacement. The utility of Lemma 7 relates particularly to those important problems where one wants to know if evidence supports either $A<\frac{N}{2}$ or $A>\frac{N}{2}$. This could very often translate into testing $\left(\frac{N}{2}-1, \frac{N}{2}+1\right)$ or 
$\left(\frac{N}{2}+1, \frac{N}{2}-1\right)$; from Lemma 7, we are assured that the power is the same in either case.

\section{TESTING $(0,1)$ OR $(1,0)$ UNDER COMPLEX SAMPLING DESIGNS}

\subsection{Testing $(0,1)$ or $(\mathbb{1}, 0)$ Under Stratified Random Sampling.}

When a finite universe is assumed to be almost or completely attribute-free (i.e., $A \approx 0$ ), any practical stratification of the universe will also yield separate strata, each of which is almost or completely attribute-free. In such cases, stratification is done mainly for sampling administrative convenience or to ensure inclusion of units in the sample from certain subuniverses or domains.

As background, assume that the finite universe of $N$ units has been partitioned into $L$ strata with $N_{i}$ units in stratum $i(i=1,2, \ldots, L)$. Let $A_{(i)}$ be the number of units with the attribute in stratum $i$; then $A=\sum_{i=1}^{L} A_{(i)}$. For testing $(0,1)$ or $(1,0)$, a stratified random sample of size $n$ is to be selected with $n_{i}$ being the size of the simple random sample from stratum $i$. To assure an optimal sampling design as defined in Section 3.3, assume proportional allocation of $n$, i.e., $n_{i}=n\left(N_{i} / N\right)$ for $i=1,2, \ldots, L$. Let $a_{i}$ be the number of units out of the $n_{i}$ sample units from stratum $i$ which have the attribute. The key statistic for inference is

$$
a_{T}=\sum_{i=1}^{L} a_{i}
$$

(Note that the usual unbiased estimator of $A$ (Cochran 1977) in this case is $\hat{A}_{s t}=$ $\sum_{i=1}^{L}\left(N_{i} / n_{i}\right) \boldsymbol{a}_{i}=(N / n) a_{T}$. $)$

If $A=\sum_{i=1}^{L} A_{(i)}=0$, the sampling distribution of $a_{T}$ is

$$
p\left(a_{T}=a_{T} \mid A=0\right)= \begin{cases}1 & \text { if } a_{T}=0 \\ 0 & \text { if } a_{T}=1,2, \ldots, n=\sum_{i=1}^{L} n_{i} .\end{cases}
$$

If $A=\sum_{i=1}^{L} A_{(i)}=1$, for some specific $j, A_{(j)}=1$ and $A_{(l)}=0$ for $l \neq j$. Thus, the sampling distribution for $a_{T}$ is given by $p\left(a_{T}=a_{T} \mid A=1\right)$ where 


$$
\begin{aligned}
p\left(a_{T}=0 \mid A=1\right) & =p\left(a_{j}=0 \mid A_{(j)}=1\right)\left\{\Pi_{l \neq j} p\left(a_{l}=0 \mid A_{(l)}=0\right)\right\} \\
& =\frac{N_{j}-n_{j}}{N_{j}}\{1\}=\frac{N-n}{N}, \\
p\left(a_{T}=1 \mid A=1\right) & =\frac{n}{N}, \text { and } \\
p\left(a_{T}=a_{T} \mid A=1\right) & =0 \quad \text { for } a_{T}=2,3,4, \ldots, n=\sum_{i=1}^{L} n_{i}
\end{aligned}
$$

Assuming $\alpha \leq(N-n) / N$ and using (13), (14), and the general form of the N-P Lemma, for testing $(0,1)$, we have

$$
\phi_{U}\left(a_{T}\right)= \begin{cases}1 & \text { if } a_{T}=1,2, \ldots, n=\sum_{i=1}^{L} n_{i} \\ \alpha & \text { if } a_{T}=0\end{cases}
$$

and for testing $(1,0)$, we have

$$
\phi_{L}\left(a_{T}\right)= \begin{cases}N \alpha /(N-n) & \text { if } a_{T}=0 \\ 0 & \text { if } a_{T}=1,2, \ldots, n=\sum_{i=1}^{L} n_{i}\end{cases}
$$

The tests in (15) and (16) under stratified random sampling with proportional allocation are the same, including in terms of power, as the corresponding tests in Section 3 under simpie random sampling. Hence, the results of Section 3 for testing $(0,1)$ or $(1,0)$ apply for stratified random sampling as described. Note that stratified random sampling with proportional allocation is an optimal sampling design because $P\left(U_{i}\right.$ selected $)=n / N$ for each $i$.

\subsection{Testing $(0,1)$ or $(1,0)$ Under Two-Stage Cluster Sampling.}

Sometimes and due to the subject matter, units in a finite universe are grouped for convenience, e.g. people living in a city are grouped by households; tires in use are grouped by vehicles; and rooms are grouped by buildings. In such cases, it is often convenient to first select a sample of the groups followed by a subselection of units from the selected groups. In this subsection, these two-stage sampling designs are considered when testing $(0,1)$ or $(1,0)$.

5.2.1 Testing with Random Selection at Both Stages. Assume that the finite universe of $N$ units has been partitioned into $M$ clusters with $N_{i}^{\prime}$ units in 
cluster $i(i=1, \ldots, M)$. Let $A_{(i)}^{\prime}$ be the number of units with the attribute in cluster $i$; then $A=\sum_{i=1}^{M} A_{(i)}^{\prime}$. To test $(0,1)$ or $(1,0)$, on the first stage a simple random sample without replacement of $m$ clusters is selected from the $M$ clusters. At the second stage and from the $i$ th sample cluster, a simple random sample without replacement of $n_{i}^{\prime}$ units is selected where $n_{i}^{\prime}=n\left(N_{i}^{\prime} / N\right)$ and $n=\sum_{i=1}^{m} n_{i}^{\prime}$ is the overall sample size. Let $a_{i}^{\prime}$ be the number of units with the attribute out of the $n_{i}^{\prime}$ sample units. Here the key statistic for inference is

$$
\sigma_{T}^{\prime}=\sum_{i=1}^{m} a_{i}^{\prime} .
$$

(Note that the usual unbiased estimator of $A$ (Cochran 1977) in this case is $\hat{A}_{C}=$ $\left.\sum_{i=1}^{m}(M / m)\left(N_{i}^{\prime} / n_{i}^{\prime}\right) a_{i}^{\prime}=(M / m)(N / n) a_{T}^{\prime} \cdot\right)$

If $A=\sum_{i=1}^{M} A_{(i)}^{\prime}=0$, then the sampling distribution for $a_{T}^{\prime}$ is

$$
p\left(a_{T}^{\prime}=a_{T}^{\prime} \mid A=0\right)= \begin{cases}1 & \text { if } a_{T}^{\prime}=0 \\ 0 & \text { if } a_{T}^{\prime}=1,2, \ldots, n=\sum_{i=1}^{m} n_{i}^{\prime}\end{cases}
$$

If $A=\sum_{i=1}^{M} A_{(i)}^{\prime}=1$, then for some specific $j, A_{(j)}^{\prime}=1$ and $A_{(l)}^{\prime}=0$ for $l \neq j$. Moreover,

$$
\begin{aligned}
p\left(\boldsymbol{a}_{T}^{\prime}=1 \mid A=1\right) & =P\left(\begin{array}{l}
j \text { th cluster is selected at stage one and the one } \\
\text { attribute is selected for its second stage sample }
\end{array}\right) \\
& =P(\text { cluster } j \text { selected }) P(\text { attribute selected / cluster } j \text { selected }) \\
& =\frac{m}{M} \cdot \frac{n_{j}^{\prime}}{N_{j}^{\prime}}=\frac{m n}{M N} .
\end{aligned}
$$

Thus, for $A=1$, the sampling distribution of $a_{T}^{\prime}$ is

$$
p\left(a_{T}^{\prime}=a_{T}^{\prime} \mid A=1\right)= \begin{cases}1-\frac{m n}{M N} & \text { if } a_{T}^{\prime}=0 \\ \frac{m n}{M N} & \text { if } a_{T}^{\prime}=1 . \\ 0 & \text { if } a_{T}^{\prime}=2,3, \ldots, n=\sum_{i=1}^{m} n_{i}^{\prime} .\end{cases}
$$

Assuming $\alpha \leq(M N-m n) / M N$ and using (18), (19), and the general form of the N-P Lemma, for testing $(0,1)$, we have 


$$
\phi_{U}\left(a_{T}^{\prime}\right)= \begin{cases}1 & \text { if } a_{T}^{\prime}=1,2, \ldots, n=\sum_{i=1}^{m} n_{i}^{\prime} \\ \alpha & \text { if } a_{T}^{\prime}=0\end{cases}
$$

with power $P\left(C_{U} \mid A=1\right)=\sum_{a_{T}^{\prime}=1}^{n} p\left(a_{T}^{\prime} \mid A=1\right)+\phi_{U}(0) p(0 \mid A=1)=\alpha+(1-$ $\alpha) \frac{m n}{M N}$; and for testing $(1,0)$, we have

$$
\phi_{L}\left(a_{T}^{\prime}\right)= \begin{cases}\frac{M N \alpha}{M N-m n} & \text { if } a_{T}^{\prime}=0 \\ 0 & \text { if } a_{T}^{\prime}=1,2, \ldots, n=\sum_{i=1}^{m} n_{i}^{\prime} .\end{cases}
$$

with power $P\left(C_{L} \mid A=0\right)=\phi_{L}(0) p(0 \mid A=0)=\frac{M N \alpha}{M N-m n}$.

Under two-stage cluster sampling with random selection at both stages and proportional allocation of $n$ at the second stage $\left(n_{i}^{\prime}=n\left(N_{i}^{\prime} / N\right)\right), P\left(U_{i}\right.$ selected $)$ $=m n / M N \neq n / N$ for each $i$; hence the design is not an optimal sampling design for testing $(0,1)$ or $(1,0)$ unless $m=M$.

\subsubsection{Testing with Probability Proportional to Size ( $\pi$ ps) Sampling}

at Stage One. It is possible, however, to define a two-stage cluster sampling design such that its power for testing either $(0,1)$ or $(1,0)$ is the same as the corresponding tests under simple random sampling or stratified random sampling as follows. On the first stage, select the $i$ th cluster without replacement with probability proportional to its size, i.e., with probability $m N_{i} / N$ for $i=1,2, \ldots, M$. At the second stage, and for the $i$ th cluster selected at the first stage, select a simple random sample where $n_{i}=n / m$ for $i=1, \ldots, m$. Here for each $U_{i}, P\left(U_{i}\right.$ selected $)$ $=\left(m N_{i} / N\right)\left(n_{i} / N_{i}\right)=n / N$; hence, the design is optimal.

Table 1 compares the power of the tests $(0,1)$ and $(1,0)$ under the four different sampling plans described in this article.

\section{EXACT RANDOMIZED UPPER CONFIDENCE BOUNDS}

Instead of testing a hypothesis as has been considered thus far in this paper, an investigator may desire to report upper confidence bounds for $A$. An observed statistic $\hat{A}_{U}$ is called a $100(1-\alpha) \%$ upper confidence bound for $A$ if for each possible value of $A, P\left(A \leq \hat{A}_{U} \mid A\right) \geq 1-\alpha$. Under simple random sampling, exact upper 
Table 1. Power Comparisons for Testing $(0,1)$ or $(1,0)$.

\begin{tabular}{|c|c|c|}
\hline \multirow[b]{2}{*}{ Sampling Design } & \multicolumn{2}{|c|}{ Power } \\
\hline & Testing $(0,1)$ & Testing $(1,0)$ \\
\hline $\begin{array}{l}\text { Simple Random Sampling } \\
\text { (Optimal) }\end{array}$ & $\alpha+(1-\alpha) \frac{n}{N}$ & $\frac{N \alpha}{N-n}$ \\
\hline \multicolumn{3}{|l|}{ Stratified Random Sampling } \\
\hline $\begin{array}{l}\text { - Proportional Allocation } \\
\text { (Optimal) }\end{array}$ & $\alpha+(1-\alpha) \frac{n}{N}$ & $\frac{N a}{N-n}$ \\
\hline \multicolumn{3}{|l|}{ Two-Stage Cluster Sampling } \\
\hline $\begin{array}{l}\text { - Random Selection Both } \\
\text { Stages/Proportional Allocation } \\
\text { at Stage Two }\end{array}$ & $\alpha+(1-\alpha) \frac{m n}{M N}$ & $\frac{M N \alpha}{M N-m n}$ \\
\hline$-\pi p s$ Selection at Stage & & \\
\hline $\begin{array}{l}\text { One/Random Selection } \\
\text { and Equal Allocation } \\
\text { at Stage Two (Optimal) }\end{array}$ & $\alpha+(1-\alpha) \frac{\mathfrak{n}}{N}$ & $\frac{N \alpha}{N-n}$ \\
\hline
\end{tabular}

bounds based on nonrandomized tests make use of the hypergeometric distribution; however, these upper bounds tend to be conservative and true coverage probabilities tend to exceed $1-\alpha$ for all values of $A$. Even when zero defectives are observed in the sample, current exact upper bounds based on nonrandomized tests can be surprisingly high (Wright 1990). In this section, we examine the construction of exact upper confidence bounds under simple random sampling based on randomized tests and note that these can lead to lower upper bounds. An important assumption throughout Section 6 is that $\alpha \leq P(a \leq A-1 \mid A)$ for each $A>0$. This very mild restriction on $\alpha$ which is very unlikely to be violated in practice, is stated here to ensure that the reported exact randomized upper confidence bound on $A$ (to be defined) is never lower than the observed value of $a$. (See Lemma 9(ii).) 


\subsection{Definition and Construction Algorithm.}

In this subsection, we give an algorithm which leads to and defines what is meant by an exact randomized upper confidence bound.

Step 1. Select a simple random sample without replacement of size $n$ from $N$ units and observe $a$ units with the attribute of interest.

Step 2. From Katz (1953), Konijn (1973), Buonaccorsi (1987), and Wright (1990, 1991), an exact nonrandomized 100(1 - $\alpha) \%$ upper confidence bound for $A$ corresponding to observed $a$ is given by

$$
\hat{A}_{U}(a)=\text { the largest value of } A \text { such that } P(a \leq a \mid A)>\alpha .
$$

Step 9. (a) For $A_{0} \geq 1$ and testing $H_{0}: A=A_{0}$ vs. $H_{1}: A<A_{0}$ at level $\alpha$, let $\phi_{L, A_{0}}$ be the exact $\alpha$-level randomized test defined on the set $\{-1,0,1,2, \ldots, n, n+1\}$; note the inclusion of -1 and $n+1$. Specifically

$$
\phi_{L, A_{0}}(a)= \begin{cases}1 & \text { if } a=-1,0,1, \ldots, a_{L}-1 \\ \frac{\alpha-\sum_{a=0}^{a_{L}-1} p\left(a \mid A_{0}\right)}{p\left(a_{L} \mid A_{0}\right)} & \text { if } a=a_{L} \\ 0 & \text { if } a=a_{L}+1, a_{L}+2, \ldots, n, n+1,\end{cases}
$$

where $a_{L}$ is such that $\sum_{a=0}^{a_{L}-1} p\left(a \mid A_{0}\right) \leq \alpha$ and $\sum_{a=0}^{a_{L}} p\left(a \mid A_{0}\right)>\alpha$.

(b) For $A_{0}=0$, take $\phi_{L, 0}(-1)=1, \phi_{L, 0}(0)=\alpha$, and $\phi_{L, 0}(a)=0$ for $a=1,2, \ldots, n, n+1$.

(c) For $A_{0}=-1$, take $\phi_{L,-1}(a)=0$ for $a=-1,0, \ldots, n, n+1$.

From (a), (b), and (c), for given $N, n$, and $\alpha$, each row of the display in Figure 1 gives the randomized test $\phi_{L, A}$ for a particular value of $A$; note the inclusion of $A=-1$ which is done only to facilitate the presentation of this algorithm.

Step 4. Thus, for observed $a$ and $\hat{A}_{U}(a)$, compute:

$$
\phi_{L, \hat{A}_{U}(a)}(a), \phi_{L, \hat{A}_{U}(a)-1}(a), \ldots, \phi_{L, 1}(a), \phi_{L, 0}(a), \phi_{L,-1}(a) .
$$

It is important to note that for $a=0,1,2, \ldots, n$,

$$
0=\phi_{L,-1}(a) \leq \phi_{L, 0}(a) \leq \phi_{L, 1}(a) \leq \ldots \leq \phi_{L, \hat{A}_{U}(a)-1}(a) \leq \phi_{L, \dot{A}_{U}(a)}(a) \leq 1
$$




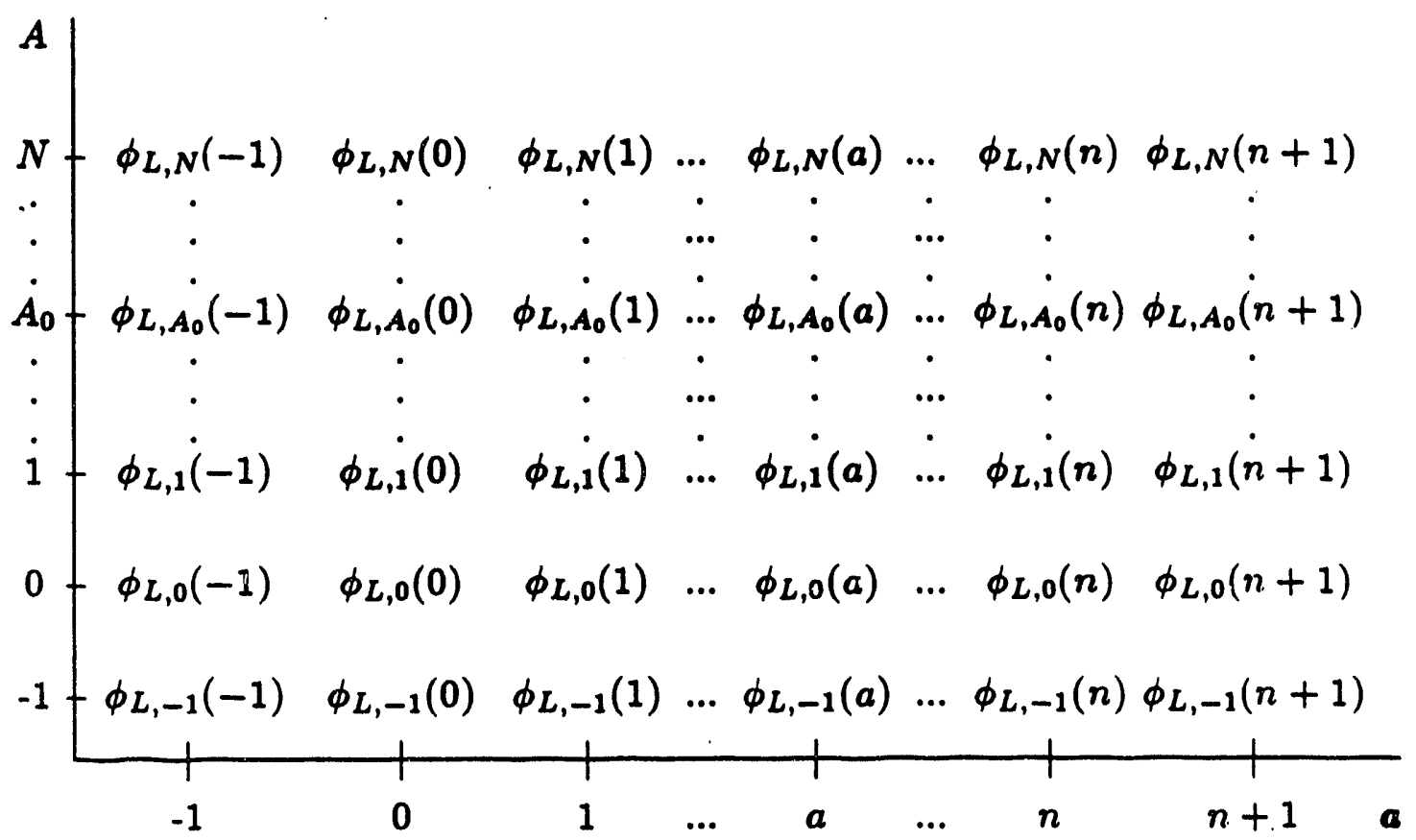

Figure 1. All Possible Values of $\phi_{L, A}(a)$.

Step 5. Randomly generate a value $r$ from $[0,1]$.

Substep 5.1. If $r \in\left(\phi_{L, \hat{A}_{U}(a)}(a), 1\right]$, take $\hat{A}_{R U}(a)=\hat{A}_{U}(a)$ and go to Step 6; otherwise, go to Substep 5.2.

Substep 5.2. If $r \in\left(\phi_{L, \hat{A}_{U}(a)-1}(a), \phi_{L, \hat{A}_{U}(a)}(a)\right]$, take $\hat{A}_{R U}(a)=\hat{A}_{U}(a)-1$ and go to Step 6; otherwise, go to Substep 5.3.

Substep 5.9. If $r \in\left(\phi_{L, \dot{A}_{U}(a)-2}(a), \phi_{L, \dot{A}_{U}(a)-1}(a)\right]$, take $\hat{A}_{R U}(a)=\hat{A}_{U}(a)-2$ and go to Step 6; otherwise, go to Substep 5.4 .

Substep 5.j. If $r \in\left(0, \phi_{L, \hat{A}_{U}(a)-(j-2)}(a)\right]$, take $\hat{A}_{R U}(a)=\hat{A}_{U}(a)-(j-1)$ and go to Step 6. If $a=0$ and $r \in\left(0, \phi_{L . \hat{A}_{U}(0)-(j-2)}\right]=(0, \alpha]$, then $\hat{A}_{R U}(0)=$ $\hat{A}_{U}(0)-(j-1)=-1$, which we take, in practice, to mean $\hat{A}_{R U}(0) \equiv 0$ if $r \in(0, \alpha]$.

Note that 5.j is the substep at which we first get an interval containing $r$ where the lower limit of the interval is 0 . 
Step 6. We say that an exact randomized $100(1-\alpha) \%$ upper confidence bound for $A$ corresponding to observed $a$ is given by $\hat{A}_{R U}(a)$. Clearly, $\hat{A}_{R U}(a) \leq \hat{A}_{U}(a)$. Also from Step 5, we see that the reported value of $\hat{A}_{R U}(a)$ depends on the observed value of $r$ as well as the observed value of $a$.

In the remainder of this section, we further discuss the sense in which we think of $\hat{A}_{R U}(a)$ as an exact randomized $100(1-\alpha) \%$ upper confidence bound for $A$ corresponding to observed $a$. In the spirit similar to that of Neyman (1934) relating to confidence intervals and discussed recently in a finite universe setting by Wright (1991), consider the set of all possible ordered pairs $(a, A)$ in the set $\{-1,0, \ldots, n, n+$ $1\} \times\{-1,0,1, \ldots, N\}$ as given in Figure 2 in the $a, A$ plane. There are $(n+3)(N+2)$ such ordered pairs.

Let $A_{0}$ be a particular value of $A$. On the straight line $A=A_{0}$, the values $a_{L}, a_{L}+1, a_{L}+2, \ldots, n-1, n, n+1$ are such that $P\left(C_{L}^{\prime}\right) \geq 1-\alpha$, where $C_{L}^{\prime}=$ $\left\{a \mid a \geq a_{L}\right\}$. Note that $a_{L}$ depends on $A_{0}$. It is important to note that for $A=A_{0}$, the set $C_{L}^{\prime}$ is the acceptance (nonrejection) region of the $\alpha$-level test $H_{0}: A=A_{0}$ vs. $H_{1}: A<A_{0}$ where $\phi_{L, A_{0}}(a)=0$ for $a=a_{L}+1, a_{L}+2, \ldots, n, n+1$ while $0 \leq \phi_{L, A_{0}}\left(a_{L}\right)<1$. Thus, $a_{L}$ may lead to rejection of $H_{0}$. Referring to Figure 2 for $A=A_{0}$, we also note that $\phi_{L, A_{0}}(a)=1$ for $a=-1,0,1, \ldots, a_{L}-1$.

For the different values of $A_{0}$, connect the $a_{L}-1$ values and call this dotted curve I; connect the $a_{L}$ values and call this dotted curve II; and connect the $a_{L}+1$ values and call this dotted curve III. In general, the ordered pairs on and above curve I have $\phi_{L, A}(a)=1$; those on and below curve III have $\phi_{L, A}(a)=0$; while the ordered pairs on curve II have $0 \leq \phi_{L, A}(a)<1$. Note that some points on curves II and III of Figure 2 might coincide. This occurs when $\sum_{a=0}^{a_{L}-1} p\left(a \mid A_{0}\right)=\alpha$.

Definition 6.1. The set of ordered pairs formed by the union of the ordered pairs on curve II with those on and below curve III is called a $1-\alpha$ randomized confidence belt, RCB.

As noted earlier, the values on and below curve III do not lead to rejection of $H_{0}$, while the values on curve II may or may not lead to rejection of $H_{0}$. 


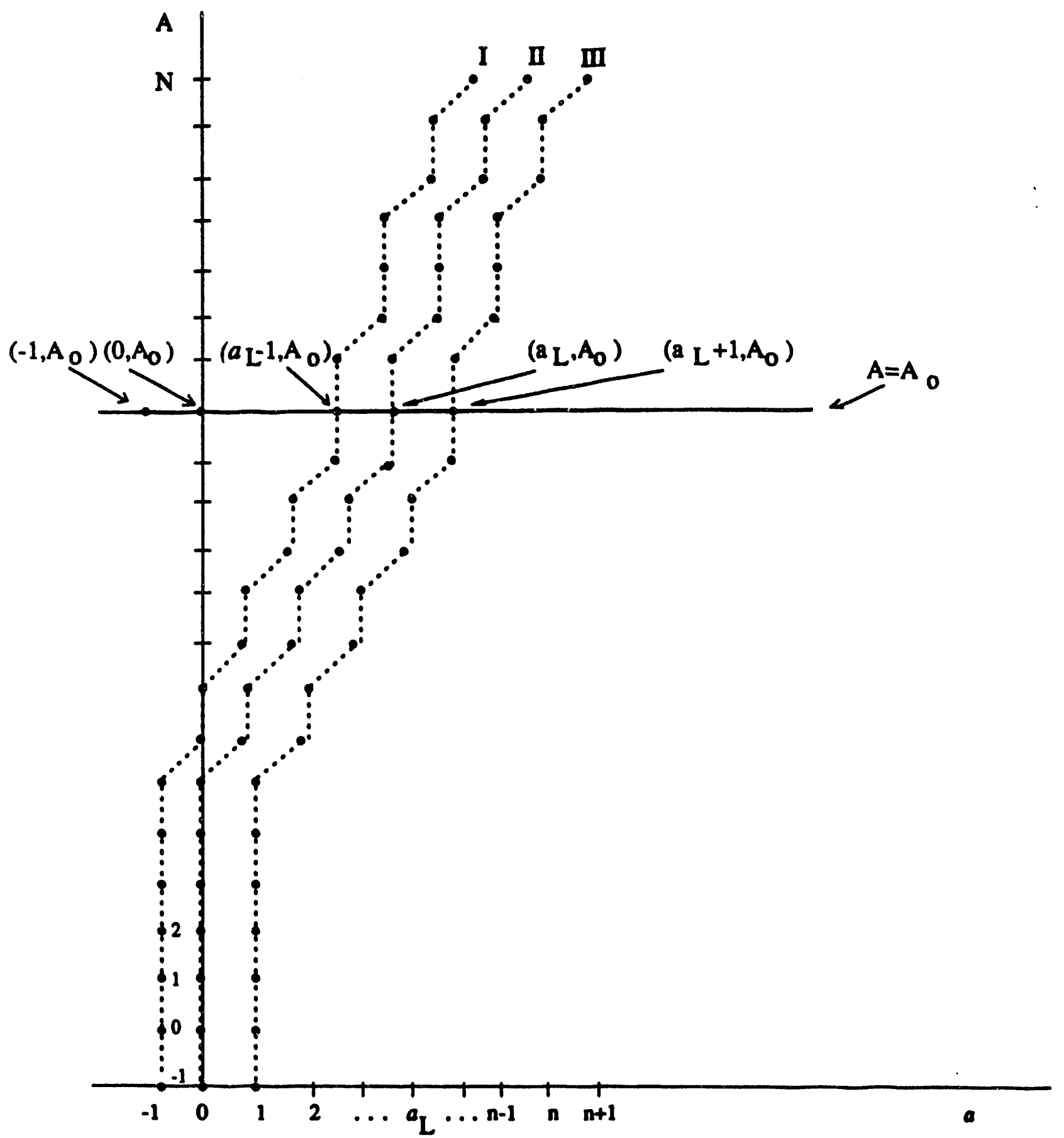

Figure 2. Curves I, II, and III 
Lemma 8. The probability that RCB does not reject $H_{0}$ is $1-\alpha$.

Proof: For each $\alpha$ and given $A, E_{A}\left[\phi_{L, A}(a)\right]=\alpha$. Hence, the probability that RCB does not reject $H_{0}$ is

$$
\begin{aligned}
P\left(\mathrm{RCB} \text { does not reject } H_{0}\right) & =E\left[1-\phi_{\dot{L}, A}(\boldsymbol{a})\right] \\
& =E\left\{E_{A}\left[1-\phi_{L, A}(\boldsymbol{a})\right]\right\} \\
& =E\left\{1-E_{A}\left[\phi_{L, A}(\boldsymbol{a})\right]\right\} \\
& =E\{1-\alpha\}=1-\alpha .
\end{aligned}
$$

Lemma 8 makes it possible to justify the construction of $\hat{A}_{R U}(a)$. For given $N, n$, and $1-\alpha$, construct a randomized confidence belt RCB as descrited above and note that the probability that RCB does not reject $H_{0}$ is $1-\alpha$ before the selection of the simple random sample. After the sample is selected and we observe $a=a$, then this additional certain information leads us to focus only on those points in the intersection of the set of points on the line $a=a$ with the set RCB as shown in Figure 3. The intersection obtained for the specific value $a$ is the set of points $\left\{(a, 0),(a, 1),(a, 2), \ldots,\left(a, \hat{A}_{U}(a)-1\right),\left(a, \hat{A}_{U}(a)\right)\right\}$. Note that $\hat{A}_{U}(a)$ is the usual exact nonrandomized $100(1-\alpha) \%$ upper confidence bound (22). Exact upper confidence bounds defined in the usual manner are conservative in that the coverage probabilities are very likely to be larger than $1-\alpha$ (Wright 1991, pp. 46-51). However, when we zandomize as described in steps 1-6 and obtain $\hat{A}_{R U}(a)$, we obtain the possibly reduced set $\left\{(a, 0),(a, 1),(a, 2), \ldots,\left(a, \hat{A}_{R U}(a)\right)\right\}$ where $\hat{A}_{R U}(a) \leq \hat{A}_{U}(a)$ and coverage probabilities for $\hat{A}_{R V}(a)$ are exactly $1-\alpha$. (See the remark after Lemma 9.)

Combining Lemma 8 and the fact that we know $a=a$, we say that possible values for $A$ are given in the set $\left\{0,1,2, \ldots, \hat{A}_{R U}(a)\right\}$ and call $\hat{A}_{R U}(a)$ an exact randomized $100(1-\alpha) \%$ upper confidence bound for $A$ corresponding to observed a because for each possible valuc of $A(>0)$,

$$
P\left(A \leq \hat{A}_{R U}(a) \mid A\right)=1-\alpha .
$$

Lemma 9 (i) $\dot{\hat{A}}_{U}(a) \geq a$ for all $a$ and $\alpha$.

(ii) $\hat{A}_{R U}(a) \geq a$ if $a>0$ and $\alpha \leq P(a \leq A-1 \mid A)$ for $A>0$. 


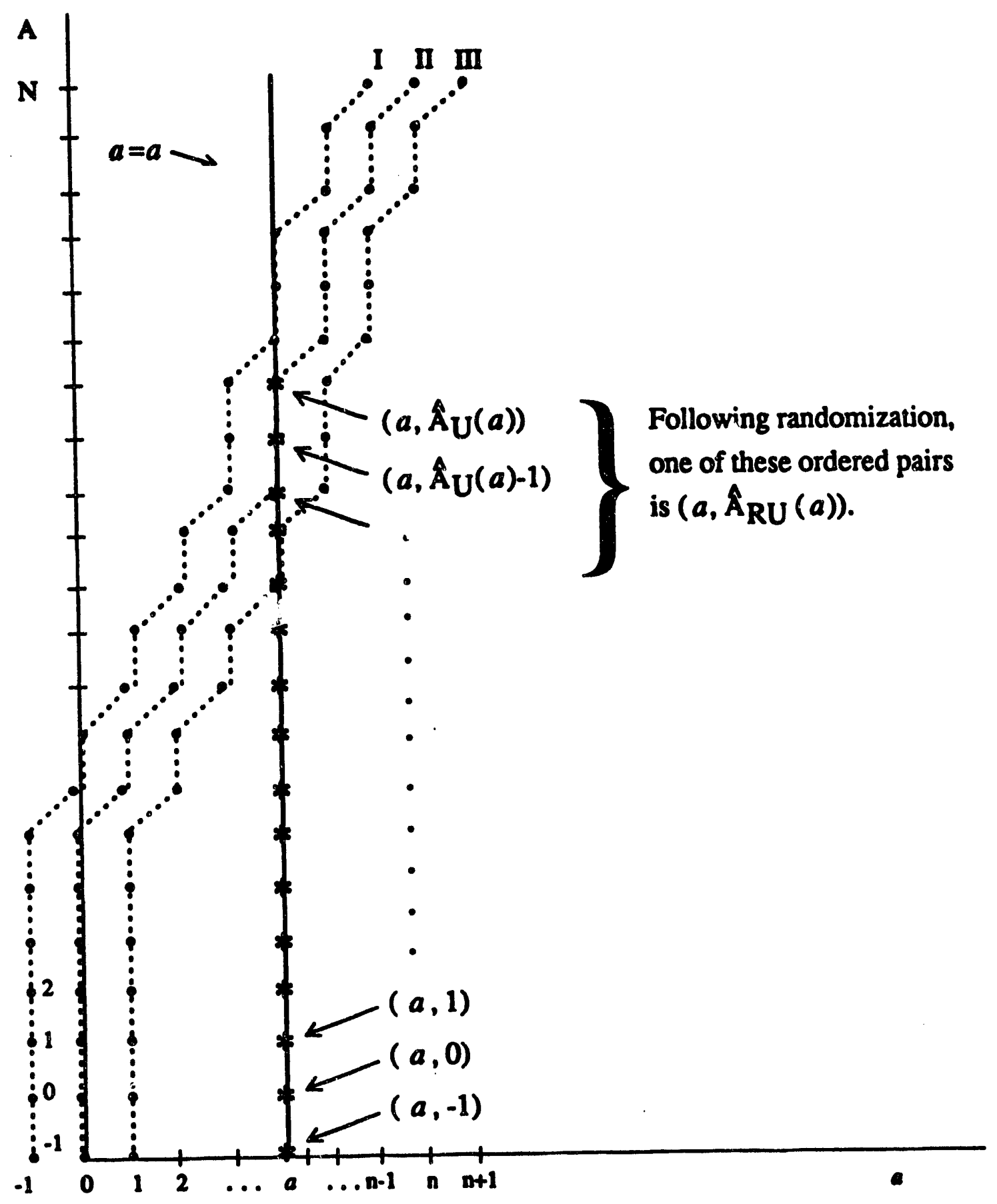

Figure 3. Visualization of an Bxact Randomized 100(1- $\alpha) \%$ Upper Confidence Bound for $A$ Corresponding to Observed a 
Proof: (i) Let $0<\alpha<1$. By (22), $\hat{A}_{U}(a)=$ the largest value of $A$ such that $P(a \leq a \mid A)>\alpha$ for observed $a$. For $A=a, P(a \leq a \mid A=a)=1>\alpha$. Thus $\hat{A}_{U}(a) \geq a$.

(ii) For $\alpha \leq P(a \leq A-1 \mid A)$ and $A>0, \hat{A}_{R U}(a)$ is defined by the algorithm in this subsection. Because $\alpha \leq P(a \leq A-1 \mid A)$, whenever $0<a=A$, we have $\phi_{L, A}(A)=0$. Thus by Step 5 and its substeps, especially $5 . \mathrm{j}, \hat{A}_{R U}(a) \geq a$ for $a>0$.

Remark on Lemma 9(ii). As noted in Substep 5.j, if $a=0$ and $r \in(0, \alpha]$, we report $\hat{A}_{R U}(0)=0$, even though the algorithm tells us that $\hat{A}_{R U}(0)=-1$. The effect of this action, in practice, is to define $\phi_{L, 0}(0) \equiv 0$ instead of $\phi_{L, 0}(0)=\alpha$. This affects Lemma 8 in that the probability that RCB does not reject $H_{0}$ is "at least" $1-\alpha$. Thus strict equality will hold in (26) for all $A$ except $A=0$ where $P\left(0 \leq \hat{A}_{R U}(\boldsymbol{a}) \mid A=0\right) \geq 1-\alpha$. An example of these coverage probabilities is given in the following example.

\subsection{Example.}

Let $N=10, n=4$, and $\dot{1}-\alpha=.975$. Figure 4 shows the possible values of $\phi_{L, A}$ in a layout similar to that in Figure 1 with curves I, II, and III as defined in Figure 2.

Thus the row for $A=6$ corresponds to the $\alpha=.025$ randomized test $\phi_{L, 6}$ of $H_{0}: A=6$ vs. $H_{1}: A<6$. We reject $H_{0}$ if $a=0$; reject $H_{0}$ if $a=1$ with probability $\phi_{L, 6}(1)=.1771$; and do not reject $H_{0}$ if $a=2,3,4$.

Assume that the desire is to provide a $97.5 \%$ upper confidence bound for $A$. Assume further that the realized value from the sample is $a=1$. Then an exact nonrandomized $97.5 \%$ upper confidence bound for $A$ is given by $\hat{A}_{U}(1)=7$. If we randomize and observe $r=.1329$, by Step 5 an exact randomized $97.5 \%$ upper confidence bound for $A$ is given by $\hat{A}_{R U}(1)=5$. Note that the coverage probabilities ior $\hat{A}_{U}(a)$ can exceed $97.5 \%$, while the coverage probabilities for $\hat{A}_{R U}(a)$ are exactly $97.5 \%$ for all $A$ except $A=0$ as noted below. Recall that Figure 4 shows $\phi_{L, 0}(0)=$ .0250 , which would lead to a coverage probability of .975 for $A=0$ if we take $A=-1$. However, in practice we would proceed as though $\phi_{L, 0}(0)=0$ because $A$ is logically never -1 , and it is this value of $\phi_{L, 0}(0)$ which is used below to obtain the coverage probability of 1 for $A=0$ in the last column of Table 2 . 


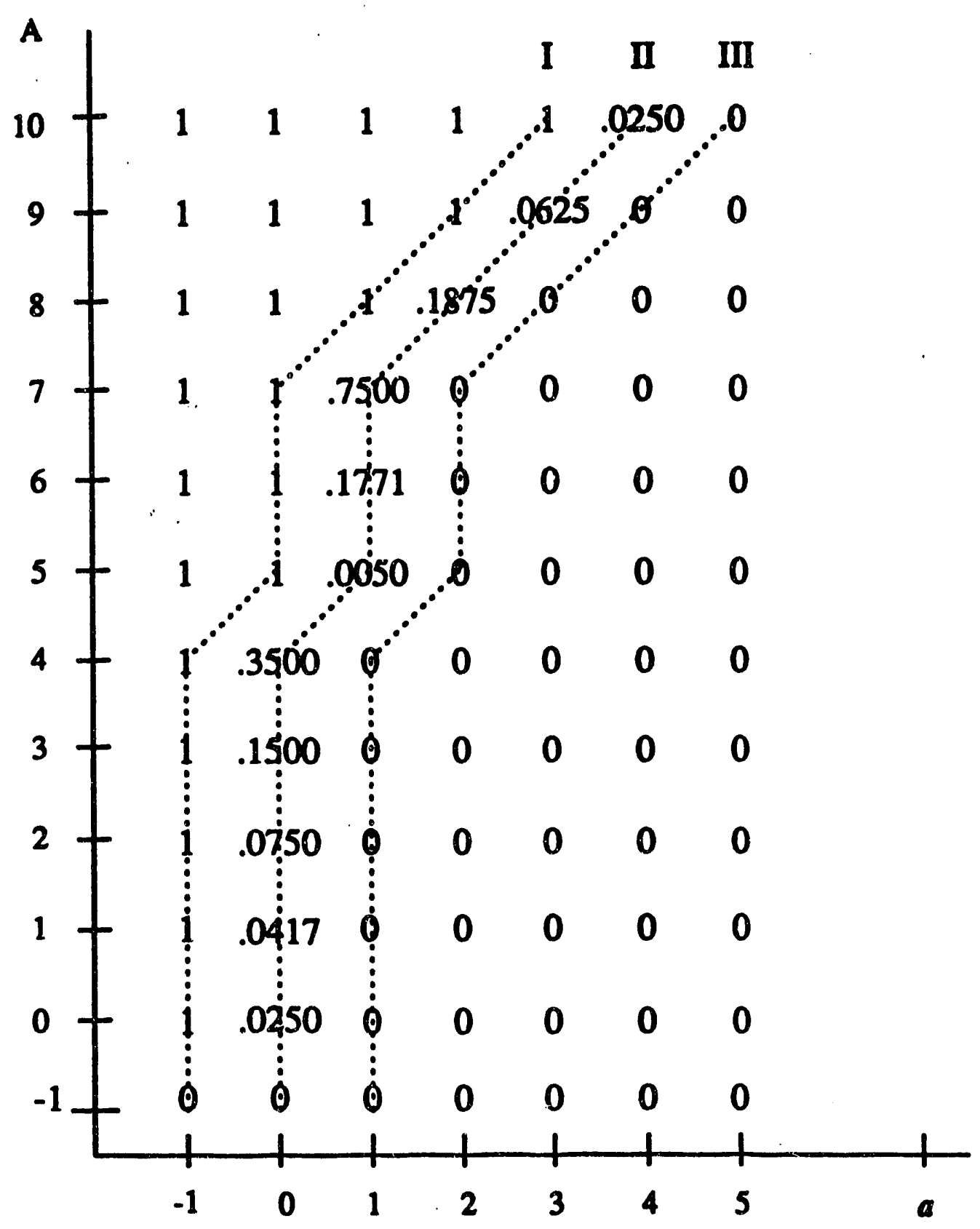

Figure 4. Vaiues of $\phi_{L, A}$ for $\hat{N}=10, n=4$, and $1-\alpha=.975$. 
Table 2. Comparison of Coverage Probabilities for $\hat{A}_{U}(\cdot)$ and $\hat{A}_{R U}(\cdot)$.

\begin{tabular}{ccc} 
Coverage Probability & $\begin{array}{c}\text { Coverage Probability } \\
\text { Using } \hat{A}_{U}(\cdot)\end{array}$ & $\begin{array}{c}\text { Using } \hat{A}_{R U}(\cdot) \\
\left(1-\phi_{L, A}(a)\right) p(a \mid A)\end{array}$ \\
$\mathrm{A}$ & $\sum_{\left\{a \mid A \leq \lambda_{U}(a)\right\}} p(a \mid A)$ & $\sum_{\left\{a \mid A \leq \lambda_{R U}(a)\right\}}$ \\
\hline 0 & 1 & 1 \\
1 & 1 & .9750 \\
2 & 1 & .9750 \\
3 & 1 & .9750 \\
4 & 1 & .9750 \\
5 & .9762 & .9750 \\
6 & .9952 & .9750 \\
7 & 1 & .9750 \\
8 & 1 & .9750 \\
9 & 1 & .9750 \\
10 & 1 & .9750 \\
\hline
\end{tabular}

\section{CONCLUSIONS}

In a continuing effort using exact methods to declare with high confidence that a universe is free of certain attributes, often referred to as defectives or errors, this article considered the question of the effect on power for specifying $A=0$ under the null hypothesis or under the alternative hypothesis where $A$ is the number of units with the attribute in a finite universe of $N$ units. Also considered is the question of constructing exact randomized upper confidence bounds on $A$ which are as tight as the usual (22) exact nonrandomized upper confidence bounds on $A$.

Under hypothesis testing and when the choice is between $A=0$ or $A=1$, it was argued that any sampling design without replacement giving equal probability of sample inclusion $n / N$ to each universe unit is optimal.

When the choice is between $A=0$ and $A=A_{1}$ where $A_{1}$ is a fixed integer $0<A_{1}<N$ and $0<\alpha \leq p\left(\boldsymbol{a}=0 \mid A_{1}\right)$ where $\boldsymbol{a}$ is the total number of units with the 
attribute in the sample of size $n$, the tests with the higher power are given below:

(i) if $0<\alpha<p\left(\alpha=0 \mid A_{1}\right) /\left\{1+p\left(a=0 \mid A_{1}\right)\right\}$, test $\left(0, A_{1}\right)$ for maximum power;

(ii) if $\alpha=p\left(a=0 \mid A_{1}\right) /\left\{1+p\left(a=0 \mid A_{1}\right)\right\}$, testing $\left(0, A_{1}\right)$ has the same power as testing $\left(A_{1}, 0\right)$; and

(iii) if $p\left(a=0 \mid A_{1}\right) /\left\{1+p\left(a=0 \mid A_{1}\right)\right\}<\alpha \leq p\left(a=0 \mid A_{1}\right)$, test $\left(A_{1}, 0\right)$

for maximum power.

When testing $\left(A_{1}, 0\right)$ where $\alpha=p\left(a=0 \mid A_{1}\right)$, the power of the test is one. Lemma 6 also gives conditions for the power of the test to be one when testing the more general case $\left(A_{1}, A_{0}\right)$. For any $\alpha$ and $n$, testing $\left(A_{0}, N-A_{0}\right)$ has the same power as testing $\left(N-A_{0}, A_{0}\right)$.

Most importantly, and contrary to current practice, this article urges that power be a major consideration when specifying the null hypothesis and the alternative hypothesis. In particular, it is clear that one may not always want to state $A=0$ as the null hypothesis. The wide availability of improved computing power makes this an easy factor to consider in practice.

On a related and important topic, we defined and presented a simple algorithm for the construction of exact randomized upper confidence bounds under simple random sampling without replacement. The clear advantage and desirable property of these randomized upper confidence bounds is that they tend to yield "tighter" upper bounds than those that we have called nonrandomized upper confidence bounds. This is especially desirable when the number in the sample with the attribute is zero. Finally, these randomized bounds have coverage probabilities exactly equal to $1-\alpha$ for all $A>0$.

\section{ACKNOWLEDGMENTS}

The author is grateful to his colleagues Charles Bayne, Kimiko Bowman, ShawPin Miaou, and Toby Mitchell who provided helpful comments and discussions which have lead to a greatly improved presentation in this paper. 


\section{REFERENCES}

1. Barabba, V. P. (1991), "Through a Glass Less Darkly," Journal of the American Statistical Association, 86, 1-8.

2. Benedict, J. C. (1990), "Comment in Letter to Editor," The American Statistician, 44, 330.

3. Bunt, L. N. H. and Barton, A. (1967), Probability and Hypothesis Testing, London: George G. Harrap \& Co. LTD.

4. Buonaccorsi, J. P. (1987), "A Note on Confidence Intervals for Proportions in Finite Populations," The American Statistician, 41, 215-218.

5. Cassel, C.-M., Särndal, C.-E., and Wretman, J. H. (1977), Foundations of Inference in Survey Sampling, New York: John Wiley.

6. Chaudhuri, A. and Vos, J. W. E. (1988), Unified Theory and Strategies of Survey Sampling, Amsterdam: North-Holland.

7. Cochran, W. G. (1977), Sampling Techniques (3rd Edition), New York: John Wiley.

8. Hajék, J. (1981), Sampling from a Finite Population, New York: Marcel Dekker.

9. Hald, A. (1981), Statistical Theory of Sampling Inspection by Attributes, London: Academic Press.

10. Hansen, M. H., Hurwitz, W. N., and Madow, W. G. (1953), Sample Survey Methods and Theory, I and II, New York: John Wiley.

11. Hwang, F. K. (1972), "A Method for Detecting all Defective Members in a Population by Group Testing," Journal of the American Statistical Association, 67, 605-608.

12. Johnson, N. L. and Kotz, S. (1977), Urn Models and Their Application, New York: John Wiley.

13. Katz, L. (1953), "Confidence Intervals for the Number Showing a Certain Characteristic in a Population when Sampling Is Without Replacement," Journal of the American Statistical Association, 48, 256-261.

14. Konijn, H. S. (1973), Statistical Theory of Sample Survey Design and Analysis, Amsterdam: North-Holland.

15. Lehmann, E. L. (1959), Testing Statistical Hypotheses, New York: John Wiley.

16. Liebermann, G. J. and Owen, D. B. (1961), Tables of the Hypergeometric Prob. ability Distribution, Stanford: Stanford University Press. 
17. Neyman, J. (1934), "On the Two Different Aspects of the Representative Method: The Method of Stratified Sampling and the Method of Purposive Selection," Journal of the Royal Statistical Society, 97, 558-606.

18. Neyman, J. and Pearson, E. S. (1933), "On the Problem of the Most Efficient Tests of Statistical Hypotheses," Philosophical Transactions of the Royal Society, Series A, 231, 289-337.

19. Odeh, R. E. and Owen, D. B. (1983), Attribute Sampling Plans, Tables, and Confidence Limits for Proportions, New York: Marcel-Dekker.

20. Panel on Nonstandard Mixtures of Distributions (1989), "Statistical Models and Analysis in Auditing," Statistical Science, 4, 2-33.

21. Raiffa, H. and Schlaifer, R. (1961), Applied Statistical Decision Theory, Boston: Division of Research, Harvard Business School.

22. Schilling, E. G. (1982), Acceptance Sampling in Quality Control, New York: Marcel-Dekker.

23. Wallenius, K. T. (1967), "Sampling for Confidence," Journal of the American Statistical Association, 62, 540-547.

24. Wright, T. (1990), "When Zero Defectives Appear in a Sample: Upper Bounds on Confidence Coefficients of Upper Bounds," The American Statistician, 44, 40-41.

25. Wright, T. (1991), Exact Confidence Bounds when Sampling from Small Finite Universes, New York: Springer-Verlag.

26. Wright, T. (1992), "A Note on Sampling to Locate Rare Defectives with Strong Prior Evidence," Biometrika, 79, 685-691.

27. Zadeh, L. A. (1965), "Fuzzy Sets," Information and Control, 8, 338-353. 
ORNL/TM-12269

\section{INTERNAL DISTRIBUTION}
1. B. R. Appleton
2. C. Bayne
3. J. J. Beauchamp
4. K. O. Bowman
5. C. C. Brandt
6. T. Darland Mathematical Sciences Lib.
7. D. J. Downing
8. D. M. Flanagan
9. E. L. Frome
10. L. J. Gray
11. T. L. Hebble
12. P. $\mathrm{Hu}$
13. E. Leach
14. W. E. Lever

15. S. -P. Miaou

16. T. J. Mitchell

17. M. D. Morris

18. G. Ostrouchov

19-23. S. Raby

24. R. L. Schmoyer

25-29. R. F. Sincovec

30-34. R. C. Ward

35-39. T. Wright

40. Central Research Library

41. K-25 Applied Technology Library

42. ORNL Patent Office

43. Y-12 Technical Library

44-45. Laboratory Records Department

46. Laboratory Records Depart. - RC

\section{EXTERNAL DISTRIBUTION}

47. Professor Roger W. Brockett (EPMD Advisory Committee), Wang Professor of Electrical Engineering and Computer Science, Division of Applied Sciences, Harvard University, Cambridge, MA 02138.

48. Dr. John P. Buonaccorsi, Mathematics and Statistics Department, University of Massachusetts, Graduate Research Tower, Amherst, MA 01003.

49. Dr. George Casella, Biometrics Unit, Cornell University, 337 Warren Hall, Ithaca, N.Y. 14853.

50. Prof. Donald J. Dudziak, Department of Nuclear Engineering Physics, North Carolina State University, Raleigh, North Carolina 27695-7909.

51. Dr. Jerome Friedman, Department of Statistics, Sequoia Hall, Stanford University, Stanford, California 94305.

52. Dr. Gerald Hahn, Mgmt. Sciences and Statistics, Corp. Research and Development Department, General Electric Company, P. O. Box 8, River Road, Schenectady, N.Y. 12301.

53. Dr. Anders Hald, Furesovej, 87 A, DK-2830 Virum, DENMARK. 
54. Dr. James E. Leiss (EPMD Advisory Committee), Rt. 2, Box 142C, Broadway, VA 22815.

55. Dr. William Q. Meeker, Statistics Department, Iowa State University, 326 Snedecor Hall, Ames, IA 50011.

56. Professor Neville Moray (EPMD Advisory Committee), Department of Mechanical and Industrial Engineering, University of Illinois, 1206 West Green Street, Urbana, IL 61801 .

57. Dr. David Nelson, Scientific Computing Staff, Applied Mathematical Sciences, Office of Energy Research, U.S. Department of Energy, Washington, D.C. 20585.

58. Dr. Robert E. Odeh, Mathematics and Statistics Department, University of Victoria, P. O. Box 3045, Victoria, V8W 3P4, CANADA.

59. Dr. Jagdiah S. Rustagi, IBM Research Center, 605/503, 5600 Cattle Road, San Jose, CA 95193.

60. Dr. Jerome Sacks, NISS, P. O. Box 14162, Research Triangle Park, North Carolina 27709-4162.

61. Dr. Edward G. Schilling, Center for Quality and Applied Statistics, Rochester Institute of Technology, One Lomb Memorial Drive, P. O. Box 9887, Rochester, N.Y. $14623-0887$.

62. Dr. L. R. Shenton, Office of Computing and Information Services, Boyd Graduate Studies Building, University of Georgia, Athens, Georgia 30602.

63. Dr. Daniel L. Solomon, Department of Statistics, North Carolina State University, P. O. Box 5457, Raleigh, North Carolina 27650.

64. Dr. Robert D. Tortora, Office of the Director, U.S. Bureau of the Census, U.S. Department of Commerce, Washington, D.C. 20233-0001.

65. Dr. How J. Tsao, Mgmt. Services Division, Eastman Kodak, Rochester, N.Y. 14652-3302.

66. Dr. Carl Wagner, Department of Mathematics, University of Tennessee, Ayres Hall, Knoxville, TN 37996-1300.

67. Dr. Ray A. Waller, S-1, Statistics, Los Alamos National Laboratory, P. O. Box 1663, Los Alamos, New Mexico 87545. 
68. Professor Mary F. Wheeler (EPMD Advisory Committee), Rice University, Department of Mathematical Sciences, P. O. Box 1892, Houston, TX 77251.

69. Dr. C. F. Jeff Wu, Department of Statistics and Actuarial Science, University of Waterloo, Waterloo, Ontario N2L3G1, Canada.

70. Professor Don Ylvisaker, Department of Mathematics, University of California, Los Angeles, California 90024.

71. Professor A. Zaslavsky, Department of Statistics, Science Center, 1 Oxford Street, Cambridge, Massachusetts 02138.

72. Office of Assistant Manager for Energy Research and Development, U.S. Department of Energy, Oak Ridge Operations nffice, P. O. Box 2001, Oak Ridge, Tennessee 37831-8600.

73-74. Office of Scientific and Technical Information, P. O. Box 62, Oak Ridge, Tennessee 37831-0062. 


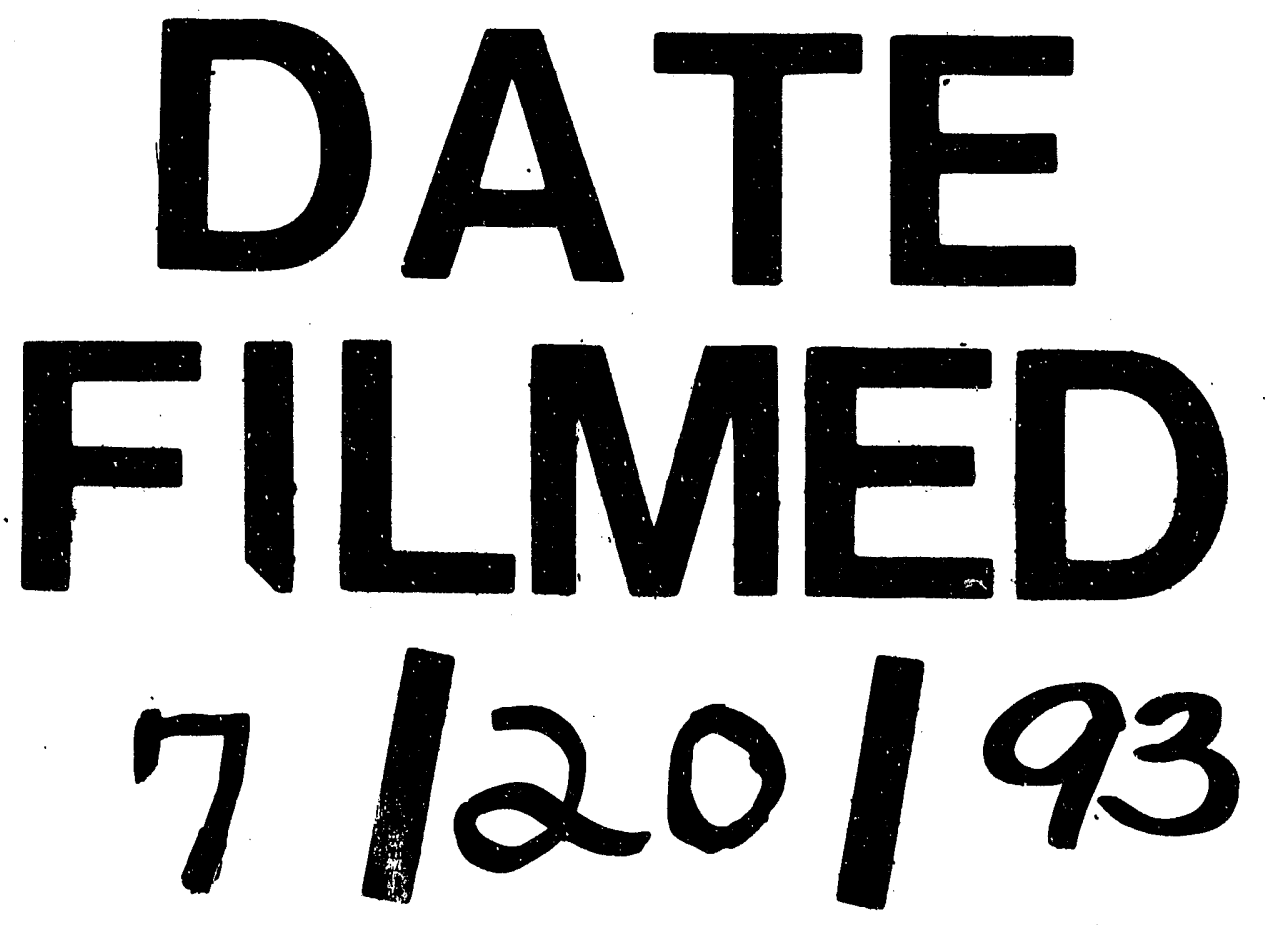


